\title{
ARTICLE
}

\section{Tectal-derived interneurons contribute to phasic and tonic inhibition in the visual thalamus}

Polona Jager ${ }^{1, \star}$, Zhiwen $\mathrm{Ye}^{2, \star}$, Xiao $\mathrm{Yu}^{2}$, Laskaro Zagoraiou ${ }^{3}$, Hong-Ting Prekop ${ }^{1}$, Juha Partanen ${ }^{4}$, Thomas M. Jessell ${ }^{5}$, William Wisden ${ }^{2,6}$, Stephen G. Brickley ${ }^{2,6}$ \& Alessio Delogu ${ }^{1}$

The release of GABA from local interneurons in the dorsal lateral geniculate nucleus (dLGN-INs) provides inhibitory control during visual processing within the thalamus. It is commonly assumed that this important class of interneurons originates from within the thalamic complex, but we now show that during early postnatal development Sox14/Otx2expressing precursor cells migrate from the dorsal midbrain to generate dLGN-INs. The unexpected extra-diencephalic origin of dLGN-INs sets them apart from GABAergic neurons of the reticular thalamic nucleus. Using optogenetics we show that at increased firing rates tectal-derived dLGN-INs generate a powerful form of tonic inhibition that regulates the gain of thalamic relay neurons through recruitment of extrasynaptic high-affinity $G A B A_{A}$ receptors. Therefore, by revising the conventional view of thalamic interneuron ontogeny we demonstrate how a previously unappreciated mesencephalic population controls thalamic relay neuron excitability.

\footnotetext{
${ }^{1}$ Department of Basic and Clinical Neuroscience, Institute of Psychiatry, Psychology and Neuroscience, King's College London, 125 Coldharbour Lane, London SE5 9NU, UK. ${ }^{2}$ Department of Life Sciences, Imperial College London, London SW7 2AZ, UK. ${ }^{3}$ Biomedical Research Foundation Academy of Athens, 4 Soranou Ephessiou Street, 11527 Athens, Greece. ${ }^{4}$ Department of Biosciences, University of Helsinki, PO Box 56, 00014 Helsinki, Finland. ${ }^{5}$ Howard Hughes Medical Institute, Columbia University, 701 West 168th Street, HHSC Room 1013, New York, New York 10032, USA. ${ }^{6}$ Centre for Neurotechnology, Imperial College London, London SW7 2AZ, UK. * These authors contributed equally to this work. Correspondence and requests for materials should be addressed to S.G.B. (email: s.brickley@imperial.ac.uk) or to A.D. (email: alessio.delogu@kcl.ac.uk).
} 
G ABAergic neurons play key roles during development, assembly and refinement of neuronal circuits ${ }^{1,2}$ before helping to shape patterns of neuronal activity in the mature brain. Whilst the development ${ }^{3-5}$ and functional diversity ${ }^{6-8}$ of GABAergic neurons have been well described in the telencephalon, less is known about the ontogeny of thalamic inhibition. Within the thalamus, GABAergic drive depends on two functionally distinct neuronal populations: intrinsic interneurons, and neurons of the reticular thalamic nucleus $(\mathrm{RTN})^{9,10}$. The origin of local thalamic interneurons in particular has remained elusive. According to the prosomeric model ${ }^{11,12}$, thalamic progenitors are specified within the second (also known as dorsal thalamus) and third (also known as ventral thalamus or prethalamus) diencephalic prosomere (p2 and $\mathrm{p} 3$ ). Inhibitory progenitors, including those of the RTN, are found in $\mathrm{p} 3$; whilst excitatory neurogenesis takes place in p2 (refs 13,14). A GABAergic rostral p2 subdomain has also been identified $(\mathrm{pTh}-\mathrm{R})^{15}$ making both $\mathrm{p} 3$ and $\mathrm{pTh}-\mathrm{R}$ possible sources of origin for thalamic interneurons.

Mapping of expression domains of key transcriptional regulators during embryonic brain development has proven instrumental for the identification of defined cell lineages. GABAergic progenitors in $\mathrm{p} 3$, including those of the RTN, express Ascl1 and progress through a differentiation programme that depends on Dlx1, Dlx2 and Arx (refs 11,16-21). GABAergic neurogenesis in pTh-R requires interaction between the pan-GABAergic proneural transcription factor Ascl1 and the GABAergic subtype-specific transcription factor $\mathrm{Helt}^{16}$. Ascll-Helt dimerization leads to sequential activation of other transcription factor genes, including Gata2, Tal1, Tal2, Gata3 and Sox14 (refs 16-19,2224), all of which are also found in the first diencephalic prosomere (p1, also known as the pretectum), but not in p3. Furthermore, reciprocal repression between $D l x 1 / 2$ and Gata $2 / 3$ suggests that alternative GABA fates are acquired in $\mathrm{p} 3$ and $\mathrm{pTh}-\mathrm{R}$ (refs 17-19). A recent report described an Otx2-positive and Sox14-negative GABAergic lineage in p3 that contributes to local thalamic interneuron ${ }^{25}$, providing experimental support to the prevailing hypothesis that dLGN interneurons have a prethalamic origin ${ }^{26,27}$. In contrast, in this study we report that $\mathrm{p} 3$, as well as pTh-R and $\mathrm{p} 1$, are unlikely sources of thalamic interneurons and propose an alternative model whereby an incoming tectal population seeds the thalamus with inhibitory interneurons in a process requiring Gata2 and Sox14.

Although interneurons are ubiquitous in the thalamus of carnivorans and primates, they are largely confined to the dorsal lateral geniculate nucleus (dLGN) in rodent ${ }^{28}$. Retinal input to the dLGN directly excites local GABAergic interneurons $(\mathrm{dLGN}-\mathrm{INs})^{29-31}$ providing visually driven feed-forward inhibition of dLGN thalamic relay neurons ${ }^{32}$. The F2 terminals formed by dLGN-INs also display atypical wiring, releasing GABA at dendro-dendritic sites ${ }^{33}$ found within triadic structures known as glomeruli ${ }^{34}$. In addition, F1 terminals are more classical axo-dendrtic synaptic arrangements. The recruitment of GABA release at the more specialized triadic structures has been shown to result in the activation of both phasic and tonic forms of inhibition in the $\mathrm{dLGN}^{35}$. In contrast, RTN neurons introduce feedback inhibition within the thalamo-cortical pathway, contributing to increased spatial attention ${ }^{36}$ and promoting synchronous activity in the thalamo-cortical axis during sleep ${ }^{37}$.

With the aim of characterizing the inhibitory properties of these dLGN-INs, we took advantage of their genetic identity and generated a Sox $14^{\text {cre }}$ knock-in mouse line to drive expression of the light-gated ion channel Channelrhodopsin 2 (ChR2). Using optogenetics we demonstrate that GABA released from Sox $14^{+}$ dLGN-INs generates a frequency-dependent form of tonic inhibition, which depends on the activation of extrasynaptic
$\mathrm{GABA}_{\mathrm{A}}$ receptors. Therefore, by exploiting the unappreciated ontogeny of dLGN-INs we have developed a novel strategy for controlling visual processing in the thalamus.

\section{Results}

Transcription factor Sox14 is a genetic marker for dLGN-INs. We and others have reported that the Sox14 gene is often associated with GABAergic neurons in subcortical brain regions ${ }^{15,17,19,22,23}$, but this association was thought not to apply for local thalamic interneurons ${ }^{25}$. The observation of scattered $\mathrm{Gfp}^{+}$cells within thalamic relay nuclei of Sox $14^{\mathrm{G} f \mathrm{p} /}+\mathrm{m}$ mice was therefore, unexpected. To investigate further the identity of these previously unreported Sox $14^{+}$neurons, we first confirmed their presence within the boundaries of the interneuron-rich dLGN visualized by anterograde labelling of the optic tract (Fig. 1a and Supplementary Movie 1) and subsequently show that they contain the neurotransmitter GABA (Fig. 1b).

To functionally classify the Sox $14^{+}$neurons, whole-cell recordings were made from $G f p^{+}$and $G f p^{-}$dLGN cells in acute brain sections prepared from 3- to 4-week-old Sox $14^{G / f p /+}$ mice (Fig. 1c). Imaging of neurobiotin fills highlighted the distinct morphology of the $\mathrm{Gfp}^{+}$neurons contrasting with the highly branched, larger dendritic coverage of the $\mathrm{Gfp}^{-}$neurons (Fig. 1d). The characteristic interneuron-like morphology of the $\mathrm{Gfp}^{+}$neurons resulted in a smaller membrane capacitance of $59.9 \pm 5.5 \mathrm{pF}(n=43)$, compared with $102.2 \pm 7.4 \mathrm{pF}(n=32)$ for the $G f p^{-}$relay neurons $(P<0.005, t$-test; Fig. 1e). The resting input resistance of $\mathrm{Gfp}^{+}$neurons $(1.1 \pm 0.1 \mathrm{G} \Omega)$ was higher $(P<0.005, t$-test $)$ than the $G f p^{-}$fraction $(0.4 \pm 0.06 \mathrm{G} \Omega)$, and the current-voltage relationship was much steeper (Fig. 1e). Analysis of the frequency of action potentials (APs) indicates lower maximum firing rates of around $20 \mathrm{~Hz}$ for the $\mathrm{Gfp}^{+}$neurons compared with $G f p^{-}$neurons. In summary, the membrane properties of the $G f p^{+}$fraction and neurotransmitter expression are compatible with the hypothesis that Sox $14^{+}$neurons are dLGN-INs, whilst all tested Gfp ${ }^{-}$cells are relay neurons ${ }^{38,39}$. However, a more direct characterization of dLGN-INs was provided by simultaneous paired recordings between the $G f^{+}$ and $\mathrm{Gfp}^{-}$neurons. In 9 out of 12 paired recordings increasing the AP firing of the $G f p^{+}$putative dLGN-IN resulted in a clear increase in the tonic conductance recorded from the $\mathrm{Gfp}^{-}$ thalamic relay neuron (Fig. 1f). The ability of a single dLGN-IN to alter the tonic conductance in a nearby thalamic relay neuron was confirmed by linear regression analysis (Fig. 1g) with $R^{2}$ values ranging from 0.3 to 0.9 (analysis of variance, $P<0.005$ ). The relationship between the firing rate of a single dLGN-IN and the increase in the tonic conductance recorded in the adjacent thalamic relay neurons was on average $31 \pm 17 \mathrm{pS} \mathrm{Hz}^{-1}(n=12)$. In contrast, the low level of direct synaptic connectivity between individual neurons meant that we did not observe a robust relationship between the rate of AP firing in a dLGN-IN and the rate of inhibitory postsynaptic currents (IPSCs) recorded in adjacent thalamic relay neurons (Fig. 1g).

Sox14 is required for dLGN-IN development. Sox14 is required for tangential migration of pTh-R GABAergic precursors between embryonic day (E) 14.5 and 16.5 (ref. 17), and its ablation results in Sox14Gfp/Gfp (Sox14 knockout) embryos having a much-reduced number of $\mathrm{Gfp}^{+}$neurons in the ventral LGN (vLGN) at the time of birth ${ }^{17}$. Because dLGN-INs are known to migrate into the nucleus postnatally ${ }^{25,26}$, we extended our previous analysis ${ }^{17}$ of the Sox 14 knockout thalamus to the postnatal brain (Fig. 2a). We found that the dLGN of P21 Sox $14^{\mathrm{G} f \mathrm{f} / \mathrm{G} f \mathrm{p}}$ mice was largely devoid of $\mathrm{Gfp}^{+}$neurons with an average of $9.3 \pm 1.0 \mathrm{Gfp}{ }^{+}$neurons in each $100 \mu \mathrm{m}$-thick section 
a

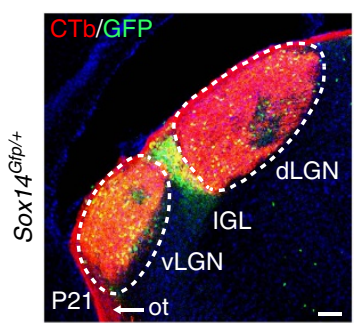

C

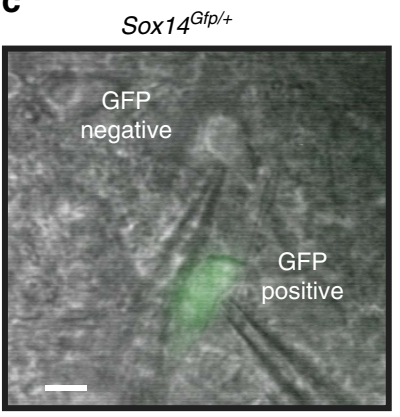

b
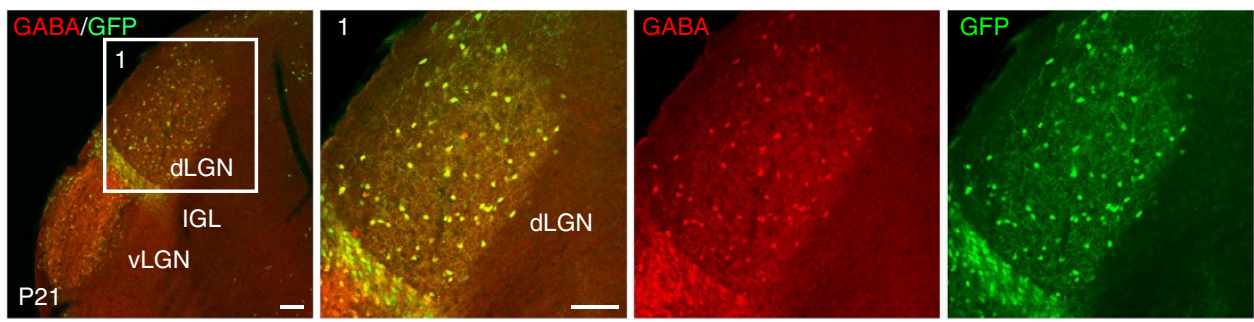

d

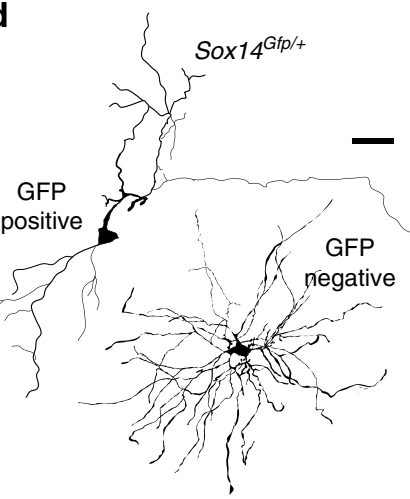

e

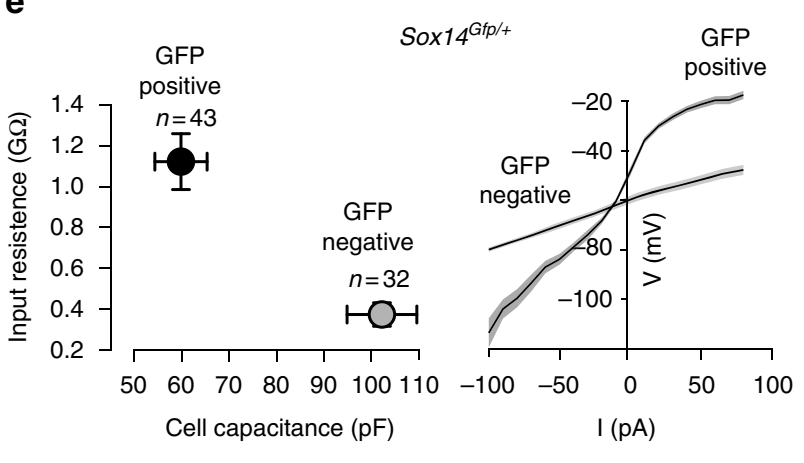

f

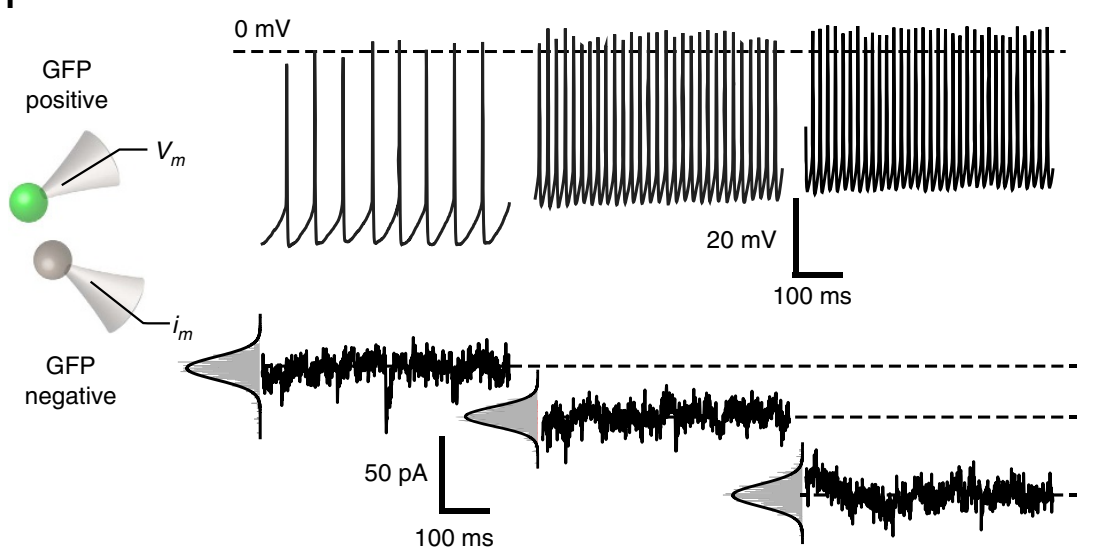

g
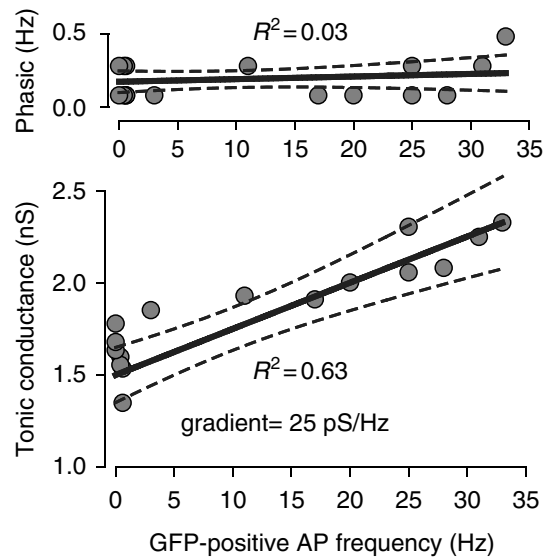

Figure 1 | Sox14 is a marker of interneurons in the dLGN. (a) Alexa-594-conjugated CTb was used to label the retinal projection to the contralateral LGN. This low-magnification image compares retinal input with the Gfp distribution in the Sox $14 \mathrm{Gfp} /+$ mouse brain at P21 3 days after injection. Scale bar, $100 \mu \mathrm{m}$ (see also Supplementary Movie 1). (b) A panel of images showing immunohistochemical detection of GABA-containing neurons in the LGN compared with the distribution of $\mathrm{Gfp}^{+}$neurons in the Sox $14^{\mathrm{Gfp} /+}$ mouse brain at P21. Scale bar, $100 \mu \mathrm{m}$. (c) Live-cell imaging data of Gfp ${ }^{+}$and $\mathrm{Gfp}^{-}$neurons in an acute slice prepared from a Sox $14 \mathrm{Gfp} /+$ mouse at P27. Patch-clamp electrodes are shown during simultaneous whole-cell recording from dLGN neurons. Scale bar, $30 \mu \mathrm{m}$. (d) Reconstructions of $\mathrm{Gfp}^{+}$and $\mathrm{Gfp}^{-}$neurons following electrophysiological recording in acute slice preparations of the Sox $14 \mathrm{Gfp} /+$ mouse. Scale bar, $50 \mu \mathrm{m}$. (e) Scatter plot of data comparing cell capacitance and input resistance in $\mathrm{Gfp}^{+}$and Gfp ${ }^{-}$neurons recorded from the dLGN of Sox $14^{\mathrm{Gfp} /}$ ${ }^{+}$mice aged between P20 and 27. Mean values are shown with error bars depicting s.e.m. Averaged current-voltage curves for these Gfp ${ }^{+}$and Gfp ${ }^{-}$ neurons is also shown. The solid lines are the average values with grey areas illustrating s.e.m. (f) Data from a simultaneous recording between a Gfp ${ }^{+}$and $\mathrm{Gfp}^{-}$neuron in an acute slice preparation of the Sox $14 \mathrm{Gfp} /+$ mouse. The voltage traces on the top were made during a whole-cell recording from a single $\mathrm{Gfp}^{+}$neuron illustrating how the action potential frequency increases at more depolarized potentials. The current traces are taken from voltage-clamp recordings made simultaneously from a neighbouring $\mathrm{Gfp}^{-}$neuron indicating the increase in tonic conductance as the presynaptic neuron increases firing rate. ( $\mathbf{g}$ ) Plot of the average relationship between the AP frequency of a Gfp ${ }^{+}$neuron and the tonic and phasic conductance changes recorded simultaneously from an adjacent $\mathrm{Gfp}^{-}$neuron. The solid lines illustrate the result of linear regression analysis with the dashed lines demonstrating $95 \%$ confidence limits of this fit.

of the dLGN (33 sections covering the entire nucleus, 3 mice) compared with $118.8 \pm 23.6$ in Sox $14^{G f p / t}$ control mice (35 sections covering the entire nucleus, 3 mice). The cell counts (Fig. 2e) indicate that Sox14 is required for the differentiation of this inhibitory subtype. Further supporting the hypothesis that Sox $14^{+}$neurons in the dLGN are the only resident inhibitory cell type, the GABA neurotransmitter and the transcript Gad1 were virtually undetectable in the dLGN of Sox14 knockout mice (Fig. 2a,b), but still present in the nearby RTN and pretectum (Fig. 2b). No increase in apoptosis was detected in Gfp-expressing regions within the LGN region between $\mathrm{P} 0$ and $\mathrm{P} 3$, suggesting that a migratory failure rather than subsequent apoptosis, leads to the observed lack of dLGNINs in the Sox14 knockout thalamus (Fig. 2c). 
a

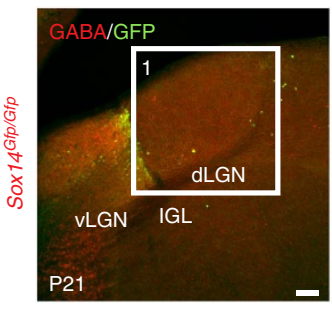

b

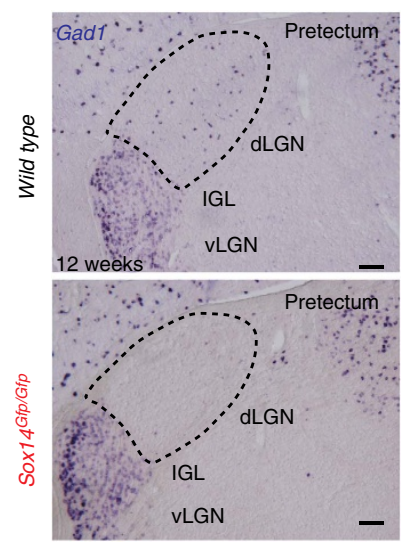

g

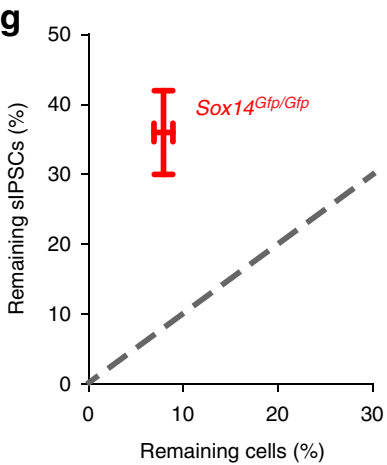

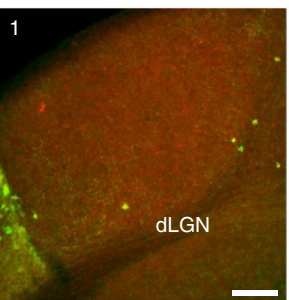

C

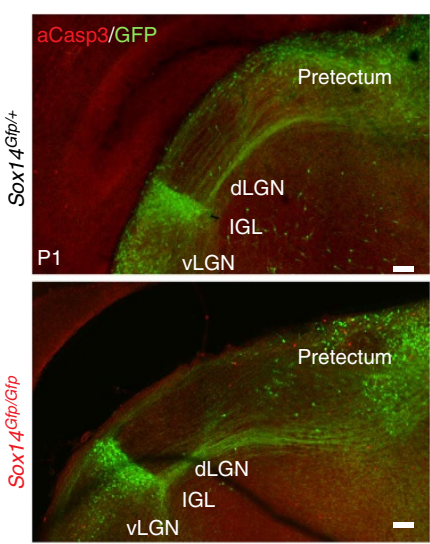

h

h $50 \mu \mathrm{M} \mathrm{DHPG}$

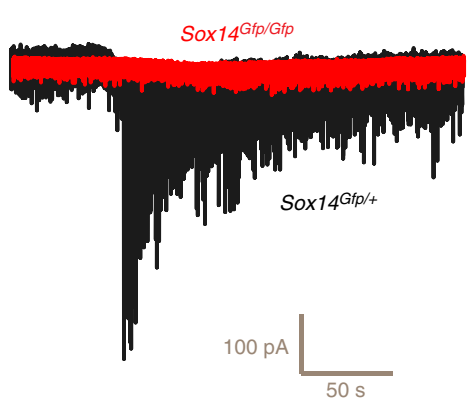

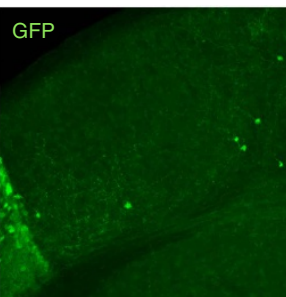

d Sox14Gtp/t

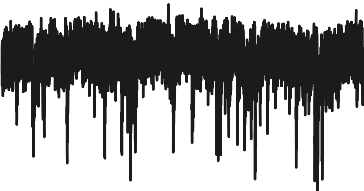

Sox14Gip/Gtp

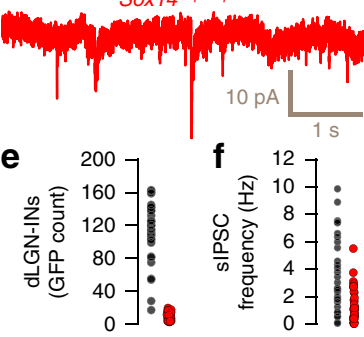

i

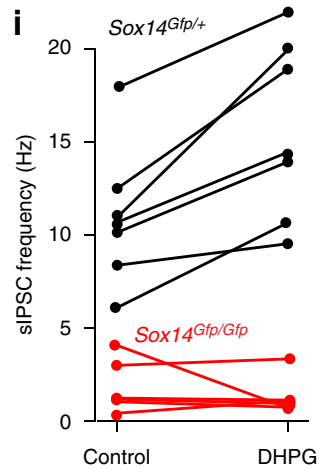

Figure 2 | Sox14 ${ }^{+}$cells provide GABAergic drive to thalamic relay neurons. (a) Panel of images taken from a knockout Sox 14 Gfp/Gfp mouse illustrating the large reduction in GABA-positive Sox14 neurons in the dLGN. Scale bar, $100 \mu \mathrm{m}$. (b) Two ISH images comparing the distribution of Gad1 mRNA (GAD67 enzyme) in a wild-type (Sox14 ${ }^{+/}$) and a Sox14 knockout (Sox14 Gfp/Gfp) mouse. Note the absence of Gad1 in the dLGN of the Sox14 knockout. Scale bar, $100 \mu \mathrm{m}$. (c) Images demonstrating similar low levels of immunolabelling for aCasp3 in the dLGN of Sox14Gfp/+ wild-type and Sox 14 Gfp/Gfp knockout brains. Scale bar, $100 \mu \mathrm{m}$. (d) Example current traces during whole-cell recordings from a relay neuron taken from a Sox14Gfp/+ wild-type or a Sox 14 Gfp/Gfp knockout mouse. (e) Scatter plots for the number of GFP-positive neurons identified in brain slices prepared from either wild-type Sox14Gfp/+ (black circles) or knockout Sox14Gfp/Gfp mice. (f) Scatter plots for the spontaneous IPSC (sIPSC) frequency measured in dLGN-INs prepared from either wild-type Sox14 Gfp/ + (black circles) or knockout Sox14 Gfp/Gfp (red circles) mice. (g) A scatter plot comparing the proportion of remaining GFP-positive cells versus the average frequency of sIPSCs recorded from relay neurons in knockout Sox 14 Gfp/Gfp mice (mean \pm s.e.m.). (h) Two superimposed current traces used to compare the impact of $50 \mu \mathrm{M}$ DHPG on tonic and phasic inhibition recorded from a wild-type Sox 14 Gfp/ + relay neuron (black trace) and a knockout Sox14 Gfp/Gfp dLGN relay neuron. Note the clear absence of any response in the knockout Sox14Gfp/Gfp dLGN relay neuron. (i) Scatter plot of the DHPGinduced change in sIPSC frequency for all relay neurons recorded from wild-type Sox $14^{G f p /+}$ (black circles) and knockout Sox 14 Gfp/Gfp mice (red circles). The solid lines link measurements made from individual relays neurons before and after application of DHPG.

Residual GABAergic drive following removal of dLGN-INs. The loss of dLGN-INs from the Sox $14^{G f p / G f p}$ mice did not result in complete removal of spontaneous IPSCs (sIPSCs) from dLGN relay neurons, most likely due to the maintenance of GABA release from RTN terminals. Nonetheless, sIPSC frequency was reduced $(P<0.005$, unpaired $t$-test $)$ from $3.6 \pm 0.5 \mathrm{~Hz}(n=31$ cells, 11 mice $)$ in Sox $14 \mathrm{Gfp} /+$ mice to $1.3 \pm 0.2 \mathrm{~Hz}(n=36$ cells, 6 mice) in Sox14 Gfp/Gfp mice (Fig. 2d). Therefore, Sox $14^{+} \mathrm{dLGN}$ INs make a significant contribution to GABAergic drive onto thalamic relay neurons (Fig. 2f), and our data could suggest that RTN terminals provide $30 \%$ of the spontaneous GABA release onto thalamic relay neurons, at least in the acute slice preparation (Fig. 2g).

To confirm that the remaining sIPSCs observed in the thalamic relay neurons of Sox $14^{G f p / G f p}$ mice originated from RTN terminals we took advantage of the fact that the F2 terminals formed by dLGN-INs are confined within the glomerular arrangement of the thalamic triad. The metabotropic glutamate receptor (mGluR) agonist (S)-3,5-dihydroxyphenylglycine (DHPG) is known to increase the rate of vesicular GABA release from dLGN-IN terminals ${ }^{35,40}$, and $50 \mu \mathrm{M}$ DHPG significantly increased $(P<0.005$, paired $t$-test $)$ the IPSC frequency from 
$10.9 \pm 1.4$ to $15.6 \pm 1.8 \mathrm{~Hz}(n=7)$ in Sox $14^{\mathrm{Gfp} /+}$ mice. However, in Sox $14^{G f p / G f p}$ mice the remaining GABA release was not affected and sIPSC frequency was $1.8 \pm 0.6 \mathrm{~Hz}(n=6)$ in control conditions compared with $1.4 \pm 0.4 \mathrm{~Hz}$ in the presence of DHPG (Fig. 2h,i) indicating a disturbance in the mGluR-mediated activation of GABA release in Sox $14^{G f p / G f p}$ mice.

dLGN-INs generate tonic inhibition in thalamic relay neurons. Having demonstrated the validity of the Sox 14 marker for identifying dLGN-INs, we then generated a novel Sox $14^{\text {cre }}$ knock-in mouse line to enable selective optogenetic activation of dLGNINs. Cre-dependent $A A V$-flex-ChR2-eYFP was injected bilaterally into the LGN of Sox14 $4^{\text {cre }}+$ mice at P16 (Fig. 3a). After 3 weeks, Chr2-eYFP-expressing cell bodies were densely concentrated throughout the intergeniculate leaflet (IGL) and were sparsely distributed throughout the dLGN with clear Chr2-eYFP in axonal terminals (Fig. 3a). To demonstrate the functional expression of ChR2 whole-cell recordings were made from dLGN-INs (Fig. 3b). In voltage-clamp configuration, $1 \mathrm{~ms}$ duration light-emitting diode (LED) pulses resulted in transient changes in the ChR2mediated conductance (Fig. $3 \mathrm{~b}$ grey trace). In current-clamp configuration, these photocurrents elicited robust APs (Fig. 3b, black trace) at LED stimulation rates of up to $30 \mathrm{~Hz}$ (Fig. 3b, blue trace). In a total of eight cells, three were clearly able to follow LED stimulation rates up to $30 \mathrm{~Hz}$, but two cells could only respond up to $20 \mathrm{~Hz}$, and three cells could only generate APs up to $10 \mathrm{~Hz}$ (Fig. 3c). However, those cells that could not follow LED frequencies above $10 \mathrm{~Hz}$ also exhibited lower firing rates in response to steady-state current injection. Moreover, steady-state somatic current injection into these dLGN-INs resulted in a linear relationship between the maximum light-evoked AP rates and the maximum AP rates observed with steady-state current injection (Fig. 3c). Next, we examined the impact of optogenetic GABA release from dLGN-INs on the excitability of the surrounding thalamic relay neurons (Fig. 3d). One millisecond light pulses delivered every second reliably evoked fast IPSCs onto dLGN relay neurons, which were blocked by gabazine (Fig. 3e). Recruitment of extrasynaptic $\delta$-GABA $\mathrm{As}$ following GABA spillover from RTN axons has been reported to prolong phasic inhibition in relay neurons of the ventrobasal complex ${ }^{41}$. A similar phenomenon occurs in the dLGN, and application of $10 \mu \mathrm{M}$ DS2, a positive allosteric modulator of $\delta$ subunitcontaining $\mathrm{GABA}_{\mathrm{A}}$ receptors $\left(\delta-\mathrm{GABA}_{\mathrm{A}} \mathrm{Rs}\right)^{43-45}$, resulted in a slowing of the ChR2-evoked IPSC that are driven by dLGN-INs (Fig. 3f). In the example shown, the evoked IPSC decay was best described by a double-exponential function. Following DS2 application the $\tau_{\text {fast }}$ component was moderately increased from 12 to $17 \mathrm{~ms}$ but the $\tau_{\text {slow }}$ component increased more markedly from 69 to $637 \mathrm{~ms}$. Across all cells there was a significant increase in the weighted decay constant $\left(\tau_{\mathrm{w}}\right)$ from $18 \pm 3(n=4)$ to $23 \pm 2 \mathrm{~ms} \quad(P<0.05$; paired $t$-test). The scatter plot (Fig. $3 \mathrm{f})$ illustrates how DS2 was capable of slowing the decay of the evoked IPSCs with no change in the $10-90 \%$ rise time.

At $1 \mathrm{~Hz}$ stimulation rates, the latency between the start of the light pulse and the evoked IPSC was $3.3 \pm 0.4 \mathrm{~ms}$, with a response probability of $0.9 \pm 0.04(n=5)$. However, at higher stimulation rates the IPSCs became less reliable (Fig. 3g). For example, following $10 \mathrm{~s}$ of stimulation at $20 \mathrm{~Hz}$ the response probability was reduced to $0.6 \pm 0.1(n=5)$. In contrast to the frequencydependent reduction in IPSC response probability, the tonic conductance was clearly enhanced at higher stimulation rates. For example, at $5 \mathrm{~Hz}$ the holding current increased by $24 \pm 7 \mathrm{pA}$ ( $n=14$ cells) compared with $172 \pm 54 \mathrm{pA}$ at $20 \mathrm{~Hz}$. The increase in holding current observed at high stimulation rates was completely abolished by application of gabazine; consistent with the presence of a tonic $\mathrm{GABA}_{\mathrm{A}}$ receptor-mediated conductance in dLGN relay neurons ${ }^{42}$. To identify the type of $G_{A B A} R$ responsible for generating this tonic conductance we applied the drug DS2. DS2 enhanced the tonic current in all cells tested $(P<0.05, t$-test, $n=4)$, and the action of DS2 on the tonic current was greater when stimulation rates were increased. In contrast, DS2 did not alter the frequency-dependent reduction in IPSC response probability (Fig. $3 \mathrm{~h}$ ). Therefore, the ability of DS2 to enhance the tonic conductance does not appear to involve any presynaptic action on vesicular GABA release, but is consistent with the action of an allosteric modulator on extrasynaptic $\delta$ $\mathrm{GABA}_{\mathrm{A}}$ Rs expressed on thalamic relay neurons.

By exploiting the Sox 14 promoter we were able to demonstrate how GABA released from the dLGN-INs results in a frequencydependent activation of extrasynaptic $\delta$-GABA $\mathrm{G}_{\mathrm{A}}$. When monitoring changes in membrane voltage in the presence of optogenetic GABA release from dLGN-INs we observed a switch from transient IPSPs at low stimulation frequencies to a steadystate hyperpolarization at higher stimulation frequencies (Fig. 3i). In all five cells examined the membrane hyperpolarized close to the estimated chloride reversal potential of $-68 \mathrm{mV}$ consistent with the activation of a tonic $\mathrm{GABA}_{\mathrm{A}}$ receptor-mediated conductance of the type shown in Fig. $3 \mathrm{~g}$.

No evidence for a thalamic origin of dLGN-INs. The discovery that Sox14 expression within the dLGN defines anatomically and functionally virtually all local interneurons implies that the current model for the ontogeny of this inhibitory cell type needs reassessing. In fact, dLGN-INs were previously hypothesized to originate in the telencephalic ganglionic eminence or ventral thalamus $(\mathrm{p} 3)^{26}$ and, more recently, described as a Otx2dependent Sox $14^{-}$lineage originating within p3 (ref. 25). We have extensively characterized neuronal precursors in the forebrain of Sox $14^{\mathrm{Gfp} /+}$ knock-in mice and could not detect any Sox $14^{+}$lineage in either of the two aforementioned territories. The presence of Sox $14^{+}$neurons in the dLGN may be explained either by incoming migration from outside the nucleus or de novo Sox14 expression. To test these hypotheses, we observed the progressive appearance of $G f p^{+}$cells in the dLGN of Sox $14^{\mathrm{Gfp} /+}$ mice between P0 and P5 (Fig. 4a) and examined their morphology. A migratory morphology could be established for $42 \%, 40 \%$ and $31 \%$ of the total $G f p^{+}$dLGN population at P0 ( $n=64$ cells), P1 $(n=211$ cells $)$ and P2 ( $n=170$ cells $)$, respectively; at subsequent days some $\mathrm{Gfp}^{+}$cells displayed morphological features of differentiated neurons, hence analysis was restricted to the $\mathrm{P} 0-\mathrm{P} 2$ window. The absence of weakly expressing $G f p^{+}$cells taken together with the abundance of migratory morphology in the $G f p^{+}$population suggests that active migration, rather than de novo activation of the Sox14 promoter, is the reason behind the appearance of $\mathrm{Gfp}^{+}$cells in the dLGN. To estimate whether directionality can be established at population level, we mapped the leading process orientation of $G \mathrm{fp}^{+}$neurons against the dLGN dorso-ventral and latero-medial axes (Fig. 4b). This was expressed both as raw angle and in its dorso-ventral and latero-medial components given by the sine and cosine, respectively (Fig. 4c). This analysis reveals that there is no obvious ventro-dorsally oriented migratory population, even at the onset of migration (P0), suggesting that a major contribution from the Th- $\mathrm{R}$ is unlikely. Instead, a predominant dorso-lateral to ventro-medial orientation of leading processes suggests a dorsal origin for dLGN-INs (Fig. 4c) $(\mathrm{P} 0: P<0.025 ; \mathrm{P} 1$ and P2: $P<10^{-3}$; one-sample Wilcoxon signed rank test).

Although the identification of a leading process and migratory morphology on fixed tissue is a good indication of on-going tangential migration, a definitive proof is the direct observation of active migration in the live tissue. Hence, we performed postnatal 
a

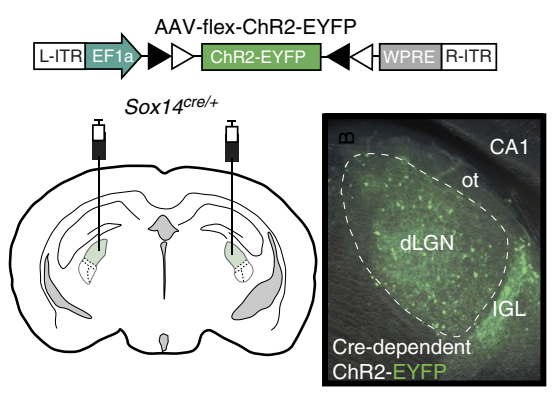

b

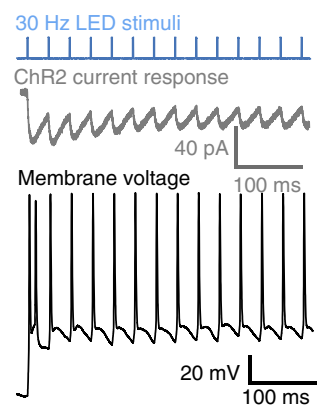

C

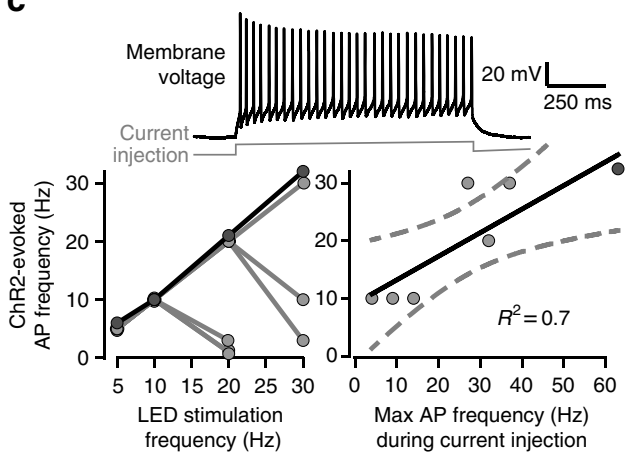

d

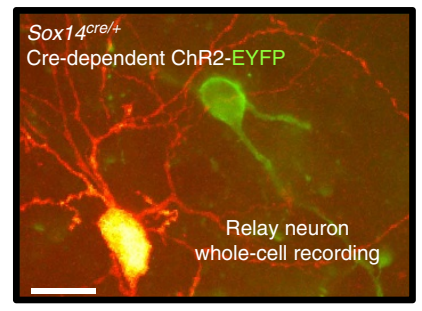

g
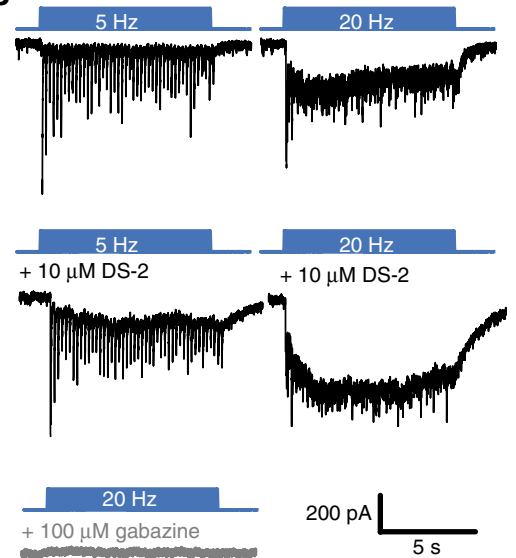

e

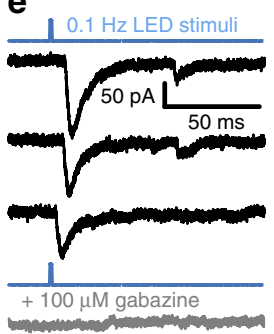

h
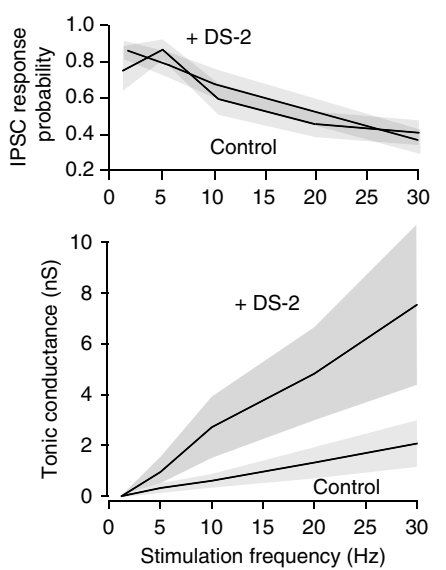

$f$
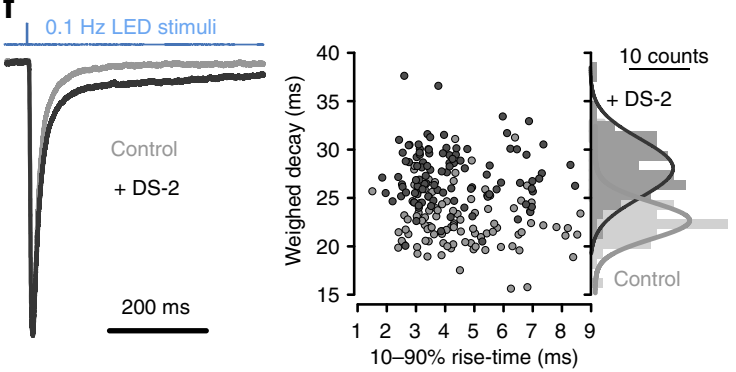

i

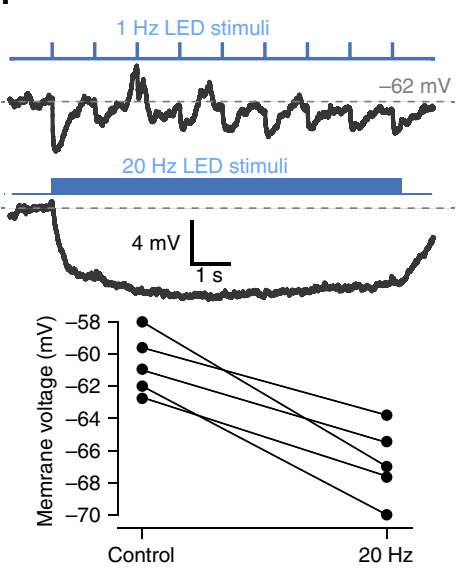

Figure 3 | Sox14 ${ }^{+}$interneurons contribute to phasic and tonic inhibition in the dLGN. (a) Flex switch strategy for delivering ChR2-EYFP into Sox14 ${ }^{\text {cre/ }+}$ neurons illustrating the injection strategy. Image of ChR2-EYFP distribution in the LGN of an acute slice preparation. (b) Voltage (black) and current traces (grey) shown in response to brief $1 \mathrm{~ms}$ blue light pulses (blue) in a ChR2-expressing Sox $14^{+}$neuron. (c) The voltage trace obtained during current injection is from the cell shown in $\mathbf{b}$. The plot on the left demonstrates the ChR2-evoked AP frequency at all LED stimulation rates with black filled symbols highlighting data from the cell shown in $\mathbf{b}$. The doublet elicited with the initial photocurrent raised the expected AP frequency for this cell. The scatter plot compares the maximum AP frequency measured during current injection with the maximum AP frequency elicited by photocurrents. (d) Image showing a ChR2-eYFP-expressing cell (green) adjacent to a filled thalamic relay neuron (red). Scale bar, $30 \mu \mathrm{m}$. (e) Current traces recorded from a thalamic relay neuron during optogenetic stimulation. The black traces show light-evoked IPSCs. The bottom grey trace is recorded with all GABA $\mathrm{A}_{\mathrm{A}}$ receptors blocked. (f) Average ChR2-evoked IPSC recorded before (grey trace) and after DS2 application (black trace). The scatter plot illustrates the relationship between IPSC rise and decay for evoked IPSCs recorded from a thalamic relay neuron. (g) The top current traces were recorded from a thalamic relay neuron during optogenetic stimulation at 5 and $20 \mathrm{~Hz}$. The current traces recorded from the same thalamic relay neuron are shown in the presence of $10 \mu \mathrm{M}$ DS2. The bottom grey trace shows the gabazine block at $20 \mathrm{~Hz}$ stimulation rates. (h) Quantification of tonic conductance and IPSC response probability in the presence and absence of DS2. The shaded areas show s.e.m. (i) Voltage trace from a thalamic relay neuron during brief light pulses (blue) delivered at 1 and $20 \mathrm{~Hz}$. Scatter plot quantifies the change in membrane voltage during $20 \mathrm{~Hz}$ optogenetic stimulation in all cells examined.

time-lapse imaging on acute coronal sections at P0.5 and monitored the movement of $\mathrm{Gfp}^{+}$neurons over a $26 \mathrm{~h}$ period ex vivo. At P0.5 the dLGN is still largely devoid of $\mathrm{Gfp}^{+}$neurons, but a stream of ventrally oriented $G f p^{+}$neurons can be found in the region immediately dorsal to the dLGN. In the course of the following $26 \mathrm{~h}$, several neurons from this region were seen entering the dLGN (Fig. 4d and Supplementary Movies 2 and 3). The migratory behaviour of $G f p^{+}$neurons in time-lapse movies correlates with higher intensity of endogenous fluorescence.
Notably, the live imaging demonstrates the presence of few Sox $14^{\text {high }}$ neurons in the IGL/vLGN and 1 of these enters the dLGN during the imaging period. This observation is consistent with the hypothesis that a small fraction of this descending population overshoots the dLGN and reaches the IGL/vLGN (Figs $6 \mathrm{~d}$ and $7 \mathrm{~d}$ ) or may subsequently refine their position and re-enter the dLGN.

In summary, the migratory morphology and behaviour of Sox $14^{+}$dLGN cells in fixed and ex vivo samples, strongly suggest 

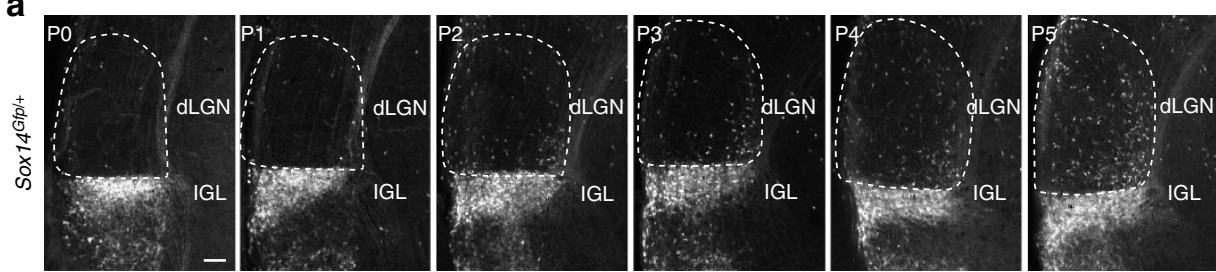

b
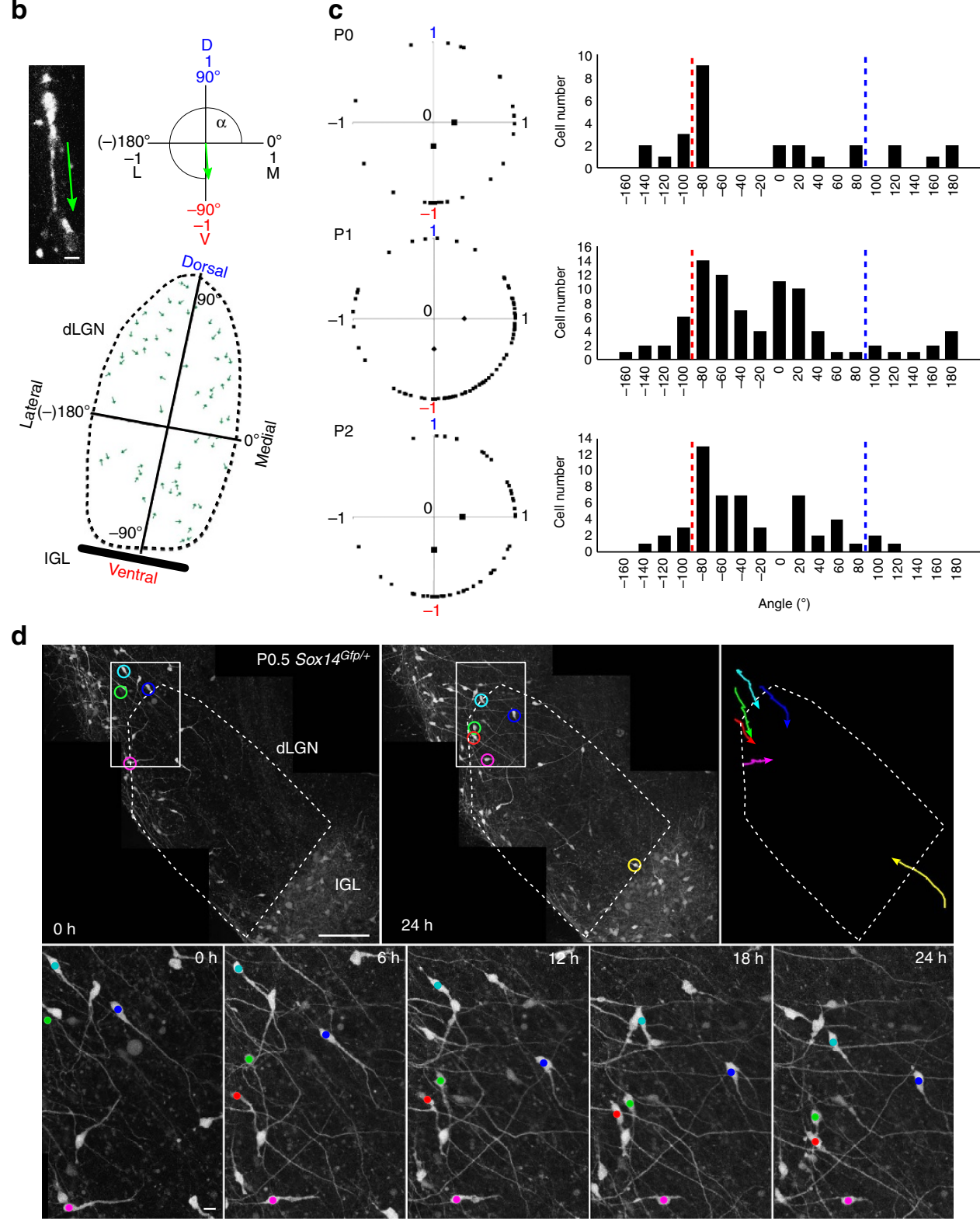

Figure 4 | Most Sox14 ${ }^{+}$neurons migrate into the dLGN in a dorsal to ventral direction. Cellular morphology and migratory behaviour of Sox14 ${ }^{+}$neurons in the fixed and ex vivo Sox $14 \mathrm{Gfp} /+\mathrm{dLGN}$. (a) Confocal imaging of Sox $14^{+}$neurons in the dLGN from PO to P5, showing the progressive spread of GABAergic interneurons in the relay dLGN. Scale bar, $100 \mu \mathrm{m}$. (b) Methodology used for population-wide analysis of the migratory morphology of Gfp ${ }^{+}$ neurons in the Sox14Gfp/+ dLGN: each green unit depicts the leading process orientation. Its angular components relative to the dorso-ventral and lateromedial axes of the dLGN can be determined. Scale bar, $10 \mu \mathrm{m}$. (c) Quantification of the orientation of leading processes at population level at P0, P1 and P2: respective histograms displaying their angular distribution in $20^{\circ}$ bins, with a maximum along the downward dorso-ventral axis (red dotted line) and lack of any obvious upwardly oriented (blue dotted line) population. Black dots on the plots of angular components are population means, which are significantly different from 0 at each developmental time point (PO: $P<0.025$; $P 2-3 P<10^{-3}$; one-sample Wilcoxon signed rank test) and indicate a dominant dorsoventral and latero-medial orientation ( $n=165$ cells from 1 brain per developmental stage). (d) Frame shots of ex vivo time-lapse imaging of an acute coronal section containing the Sox14 Gfp/ $+\mathrm{dLGN}$ at P0.5 and imaged over the following $26 \mathrm{~h}$. Neurons that cross the boundaries of the dLGN are colour coded post acquisition for clarity (Supplementary Movies 2 and 3). Scale bar, $100 \mu \mathrm{m}$; and $10 \mu \mathrm{m}$ for close-up images. 
a

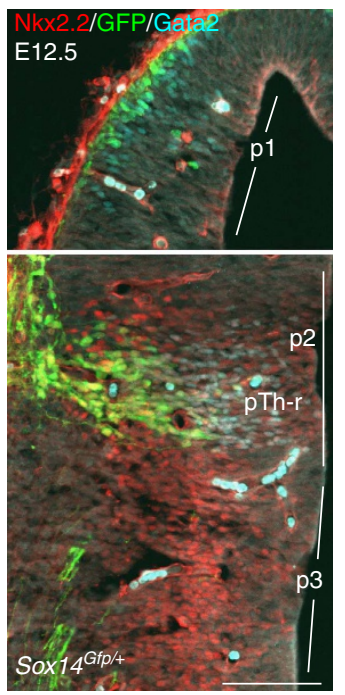

b

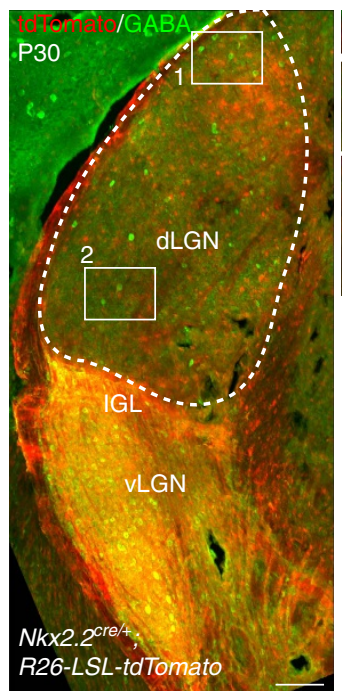

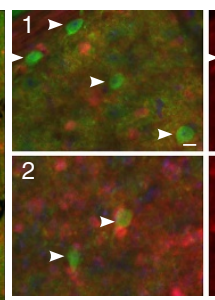

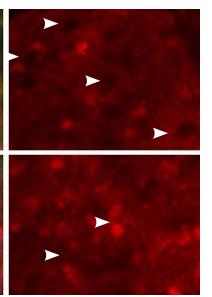

C
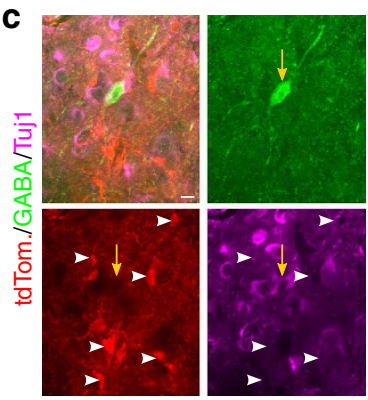

Figure 5 | Fate mapping of $\mathbf{N k x 2 . 2}$ progenitors in the dLGN excludes GABA interneurons. Nkx2.2 $2^{c r e /+; R 26-L S L-t d T o m a t o}$ conditional reporter expression was used to trace the fate of Nkx2.2 progenitors in the thalamus. (a) IHC in the E12.5 brain of a Sox 14 Gfp/ + embryo shows Nkx2.2 in progenitors of p3 and in the pTh-R domain of $\mathrm{p} 2$, which together define the presumptive GABA-rich vLGN and IGL territory, respectively (see also $\mathbf{b}$ ). Nkx2.2 progenitors are not present in p1. Sequential activation of Gata2 and Sox14 defines a subset of Nkx2.2 progenitors in the pTh-R domain. Scale bar, $100 \mu$ m. (b) Adult brains

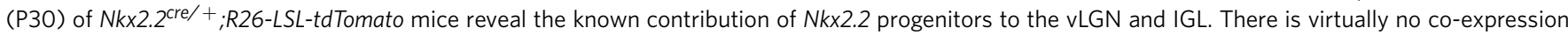
of GABA and tdTomato in the dLGN (white arrowheads in insets 1 and 2; out of 586 GABA ${ }^{+}$dLGN cells counted, 576 are negative for tdTomato and only 10 may have weak or unclassifiable tdTomato expression; $N=3$ brains). Scale bars are $100 \mu \mathrm{m}$ in the low-magnification image and $1 \mu \mathrm{m}$ in insets. (c) The fate of $N k \times 2.2$ progenitors in the dLGN is largely non-neuronal, as indicated by weak to no staining for the pan-neuronal marker Tuj 1 in tdTomato ${ }^{+}$cells of

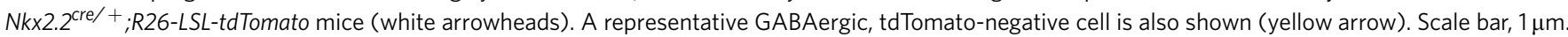

that interneuron precursors reside in a dorsal region before entering the dLGN by active migration.

To conclusively rule out any contribution to dLGN-INs from p3 and p2 we took advantage of the expression of the $N k \times 2.2$ gene, which labels GABAergic fates in the presumptive vLGN and IGL $^{15,22}$ (Fig. 5a,b), to fate map $N k \times 2.2$ lineages with the previously described $N k x 2.2^{c r e /+}$ knock-in line ${ }^{46}$. Fate mapping in adult brains from $N k x 2.2^{\text {cre }} ; R 26-L S L$-tdTomato mice reveals that, within the dLGN, the $\mathrm{GABA}^{+}$and the tdTomato ${ }^{+}$populations are mutually exclusive (Fig. 5b,c), with $98 \%$ of GABA ${ }^{+}$ cells in the dLGN negative for tdTomato $(n=586$ cells from 3 brains). Most tdTomato ${ }^{+}$cells are $\mathrm{TuJ1}^{-}$(Fig. 5c) and likely glia, in agreement with the known function of $N k x 2.2$ in oligodendrocyte differentiation ${ }^{47}$, as well as the role in neurogenesis. The evidence obtained so far is not supportive of a $\mathrm{p} 3$ or $\mathrm{p} 2$ origin for dLGN-INs, pointing instead at Sox $14^{+}$and Nkx $2.2^{-}$dorsal-caudal structures such as the pretectum (p1) and dorsal midbrain (Figs 5a, 7f).

dLGN-INs are a Sox14 ${ }^{+}$Otx2 ${ }^{+}$lineage. Conditional mutagenesis has shown that $\mathrm{Otx} 2$ is required at the onset of tangential migration (P0) for the specification of dLGN-INs and that the dLGN of Otx2-ablated mice is devoid of virtually all inhibitory neurons ${ }^{25}$. We have therefore reasoned that the subpopulation of Sox $14^{+}$precursors that gives rise to dLGN-INs must also be $\mathrm{Ot} \times 2^{+}$. Before the onset of tangential migration (E17.5), Otx $2^{+}$ neurons are present in the prethalamic side of the $\mathrm{p} 3 / \mathrm{p} 2$ border, in the pretectum (p1) and in the adjacent superior colliculus (SC), but co-expression with Sox14 is observed only within the superior colliculus and in isolated cells lining the pretectum and dLGN (arrows) (Fig. 6a). By the time dLGN-INs enter the dLGN (P3), Sox $14^{+}$Ot $x 2^{+}$double-positive neurons can be seen in the dLGN (Fig. 6b,d), in the superior colliculus and also forming a narrow stream along the intervening outer region of the pretectum (arrows in Fig. 6c). The presence of a cluster of Sox $14^{+} \mathrm{Ot} \times 2^{+}$ double-positive precursors in the superior colliculus just before birth that extends towards the dLGN is suggestive of a tectal origin for dLGN-INs. Before the onset of tangential migration (E17.5) the pretectum contains many Otx $2^{+}$neurons, but none is also Sox $14^{+}$, suggesting that $\mathrm{p} 1$ is an unlikely source of So $14^{+}$Ot $x 2^{+}$double-positive dLGN-INs. Although there was no obvious Sox $14^{+}$Otx $2^{+}$double-positive population in the immature vLGN (E17.5-P3), some isolated double-positive cells were observed there at P3 (Fig. 6d,b), these may be migrating dLGN-INs that have overshot the dLGN and entered the vLGN (Fig. 7d). In summary, expression and loss-of-function data for Otx2 and Sox 14 help to refine the ontogeny of dLGN-INs to a Sox $14^{+}$Ot $x 2^{+}$double-positive precursor subtype within the largely non-overlapping domains of Otx2 ${ }^{+}$and Sox $14^{+}$cells, mapping the likely location of dLGN-IN precursors to the superior colliculus (Fig. 6e).

Tectal Sox14 precursors migrate into the dLGN. To directly test the possibility that Sox $14^{+}$interneurons migrate into the dLGN via a dorso-caudal to ventral route from the superior colliculus, we performed in utero labelling of Sox $14^{+}$tectal precursor cells. We have injected through the uterine wall directly in the superior colliculus of E15.5 Sox14 $14^{\text {cre/ }}+$ embryos a cre-dependent AAV expressing a red fluorescent protein (EF1a-flex-tdTomato). The dorso-caudal end of the telencephalic folds was taken as a landmark and the needle inserted just caudal to that. We then examined the location of labelled cells in juvenile mice at P14 (Fig. 7e). As expected, the injection site in the superior colliculus exhibited strong fluorescent signal (Fig. 7a), but no fluorescent labelling could be detected in more rostral pretectal territory, where a high number of Sox $14^{+}$neurons reside (Fig. 7b,c). 
a
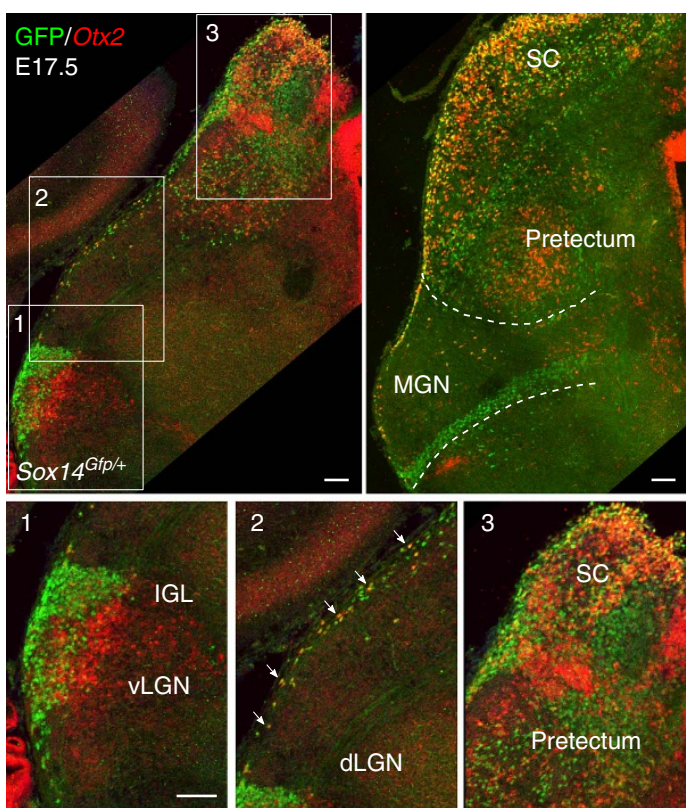

e

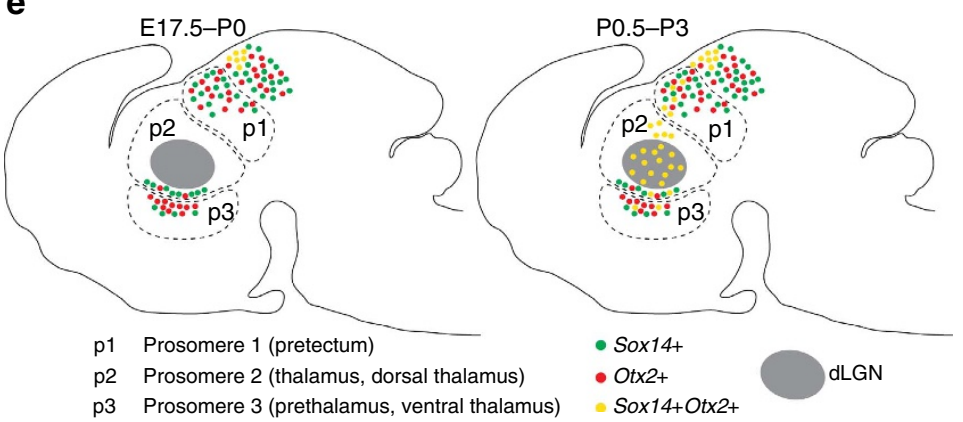

p3 Prosomere 3 (prethalamus, ventral thalamus) Sox14+Otx2+ b

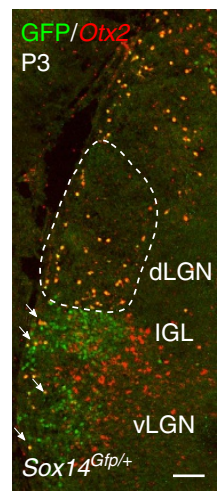

d

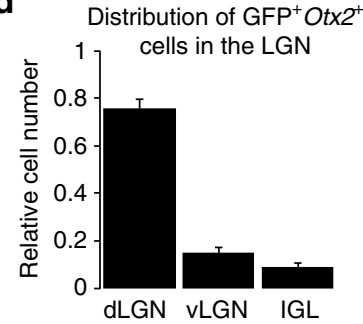

c
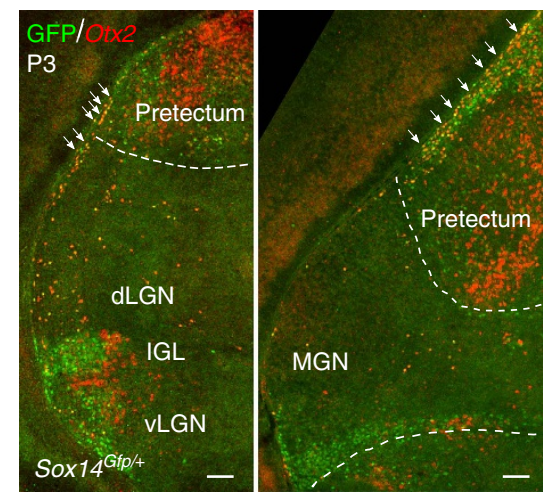

GFP and Otx2

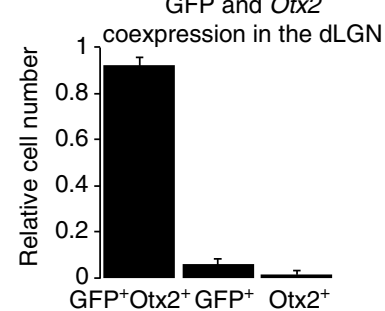

Figure 6 | Sox14 and Otx2 are co-expressed in the embryonic tectum and both define the dLGN-IN population. ISH for Otx2 and IHC for GFP showing the location of double-positive Sox $14{ }^{+}$Otx $2^{+}$cells before (E17.5) and after (P3) birth in Sox14 Gfp/+ brains. (a) At E17.5 Gfp ${ }^{+}$and Otx2 ${ }^{+}$neurons occupy adjacent domains of the vLGN (inset 1) and of the pretectum (inset 3). Sox14 ${ }^{+}$Otx2 ${ }^{+}$double-positive neurons are present in the outermost region of the superior colliculus (SC) and forming a stream of cells that extends from the SC towards the dLGN along the surface of the brain (white arrows in inset 2). (b) At P3 Sox $14^{+} \mathrm{Ot} \times 2^{+}$double-positive neurons are clearly visible in the dLGN and scattered in other thalamic nuclei. Furthermore, some double-positive neurons are also present in the IGL and vLGN (white arrows) and quantified in $\mathbf{d}$. (c) The stream of Sox $14^{+}$Otx2 ${ }^{+}$double-positive neurons lining the brain at the level of pretectum and thalamus is highlighted with white arrows. (d) Quantification of the relative distribution of Sox14 ${ }^{+} \mathrm{Otx}^{+}{ }^{+}$double-positive neurons in the three domains of the LGN (dLGN: 75.96 \pm 3.71 ; vLGN: $14.93 \pm 2.18$; IGL: $9.10 \pm 1.75$; \%, mean \pm s.e.m.; $n=531$ cells from 3 brains) and the relative numbers of double-positive and single-positive neurons in the dLGN (Gfp ${ }^{+} \mathrm{Otx}^{+}: 92.15 \pm 0.47 ; \mathrm{Gfp}^{+}: 6.23 \pm 0.36$; Otx2 ${ }^{+}: 1.62 \pm 0.81 ; \%$, mean \pm s.e.; $n=651$ cells from 3 brains). (e) Schematic summary of the distribution of Sox $14^{+}$Ot $\times 2^{+}$double-positive and single-positive neurons across the thalamic (p3 and p2), pretectal (p1) and SC territories, before and after birth. Scale bars, $100 \mu \mathrm{m}$.

Instead, and in agreement with our hypothesis, the dLGN contained scattered labelled cells (Fig. 7b). Higher-resolution imaging revealed their polar morphology, consistent with them being inhibitory interneurons (Fig. 7b).

Importantly, at the time of injection and until birth, the dLGN is devoid of Sox $14^{+}$cells, hence the fluorescently labelled neurons present in the nucleus at P14 must have entered it via tangential migration from the injected area. The possibility that the injected AAV solution may have spread within the extracellular space to reach the thalamus can be ruled out as no labelled cells were observed in Sox $14^{+}$regions located between the injection site and the thalamus, such as the anterior pretectum (Fig. 7b,c). Therefore, we conclude that Sox14 precursor cells migrating from the dorsal midbrain are the source of dLGN-INs.

Reminiscent of the few Otx $2^{+}$Sox $14^{+}$neurons observed in the IGL/vLGN (Fig. 6d), we have found some tdTomato-labelled tectal neurons reaching the vLGN ( $<20 \%$ of total tdTomatolabelled LGN cells; $n=145$ ). The high degree of correlation $\left(R^{2}=0.98\right.$; Fig. $\left.7 \mathrm{~d}\right)$ in the distribution of tectal-derived Sox14 neurons across the LGN subdomains that have been either labelled by Sox14Otx2 co-expression or by cre-dependent AAV infection, further supports the conclusion that double-positive Ot $x 2^{+}$Sox $14^{+}$precursors reside in the dorsal midbrain before birth and migrate ventrally to seed the dLGN with local interneurons. Whilst most neurons terminate their migration in the dLGN, a smaller fraction continues migrating to reach the vLGN.

Sox14 is not expressed in neuronal progenitors, hence the remote possibility exists whereby dLGN-IN precursors reside transiently in the dorsal midbrain between E15.5 and the time of birth, but are actually born elsewhere. To test whether dorsal midbrain progenitors contribute to dLGN-INs, we first 
a

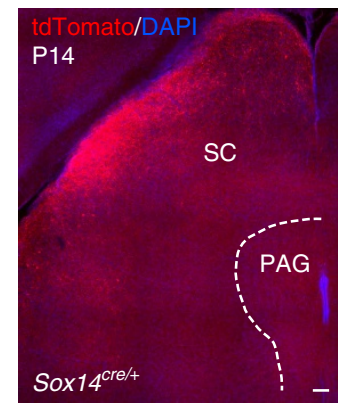

b
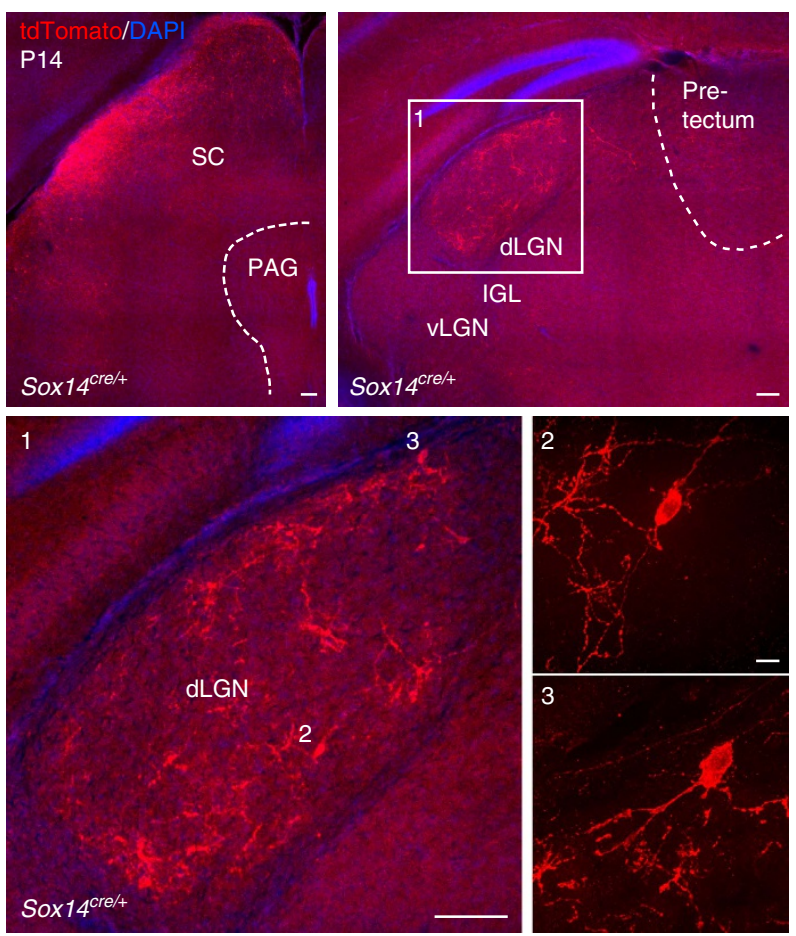

f
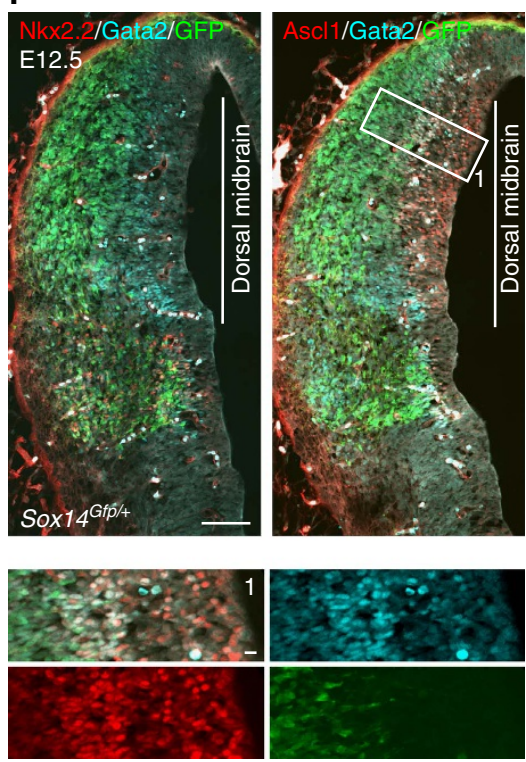

C

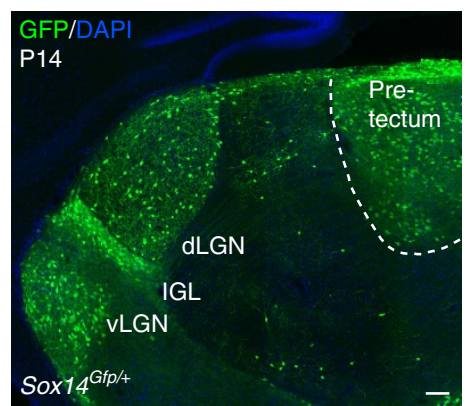

d Correlation of relative distribution in LGN

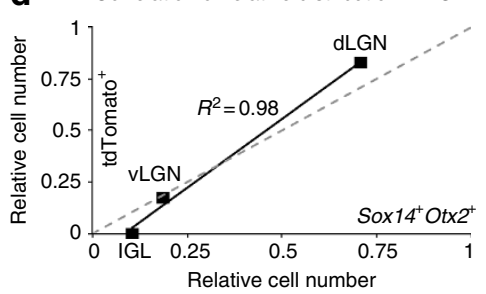

e
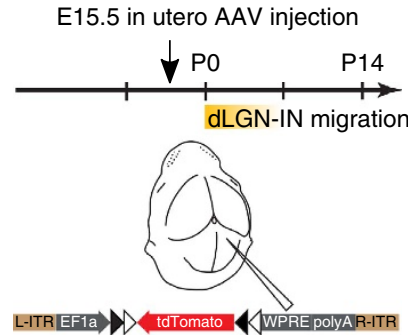

g
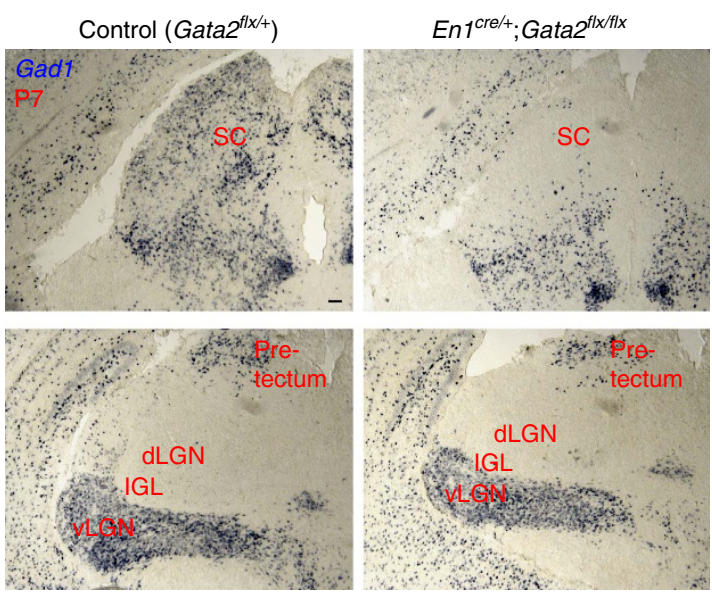

Figure 7 | Fate mapping of tectal Sox14 ${ }^{+}$precursors. Labelling of Sox $14^{+}$precursors via in utero focal injection of $A A V 2 / 1-E F 1 \alpha-D / O-t d T o m a t o ~ v i r u s$ into the SC of Sox14 ${ }^{\text {cre/ }+}$ embryos at E15.5. The time line of the experiment is summarized in e. (a) At P14, the cre-dependent expression of tdTomato labels the site of injection in the outer-most region of the left SC. Scale bar, $100 \mu \mathrm{m}$. (b) Coronal sections at thalamic level reveal an enrichment of tdTomatoexpressing cells within the boundaries of the dLGN. Scale bars, $100 \mu \mathrm{m}$. Higher-magnification confocal imaging (insets 2 and 3 ) reveals the typical morphology of local Ins; scale bar, $10 \mu \mathrm{m}$. (c) The location of Sox $14^{+}$neurons in a comparative section from an age-matched Sox 14 Gfp/ + brain illustrates the expected labelling pattern if the injected AAV-containing solution had passively spread from the site of injection to the thalamus. Scale bar, $100 \mu \mathrm{m}$. (d) Although not visible in $\mathbf{b}$, in some brains tdTomato-labelled cells could be detected in the vLGN. The distribution of tdTomato-labelled Sox $14{ }^{+}$tectal precursors in the LGN subdomains is highly correlated to that of Sox14 ${ }^{+}$Otx2 ${ }^{+}$neurons in the same regions $\left(R^{2}=0.98\right)$. (e) Time line of the in utero $\mathrm{AAV}$ injection and indicative view of the morphological landmarks used to select the unilateral injection site on the SC. (f) Neurogenesis in the E12.5 Sox14Gfp/ + dorsal midbrain displaying the sequential activation of Ascl1 (Mash1), Gata2 and Sox14 (also see inset 1), and lack of Nkx2.2, which is instead expressed in a Sox $14^{+}$ventral midbrain domain. Scale bars, $100 \mu \mathrm{m}$, and $10 \mu \mathrm{m}$ for the inset. (g) Detection of the Gad1 transcript in P7 control and Gata2 conditional knockout brains. The En $7^{\mathrm{cr} /}+\mathrm{d}$ driver selectively recombines the floxed ( $\mathrm{flx}$ ) Gata2 allele in the midbrain, but not in more rostral forebrain structures. The marked reduction of $\mathrm{Gad1}^{+}$neurons in the SC is accompanied by a similarly strong reduction of Gad1 ${ }^{+}$cells in the dLGN. In contrast, the density of Gad1 ${ }^{+}$ neurons in the intervening pretectum, as well as vLGN/IGL remains grossly normal. Scale bar, $100 \mu \mathrm{m}$. 
established the time of dLGN-IN genesis via 5-bromo-2'deoxyuridine $(\mathrm{BrdU})$ incorporation into S-phase genomic DNA of proliferating neural stem cells over the E10.5-E13.5 window of embryonic development, in Sox $14^{\mathrm{Gfp} /+}$ mice. This birth-dating experiment revealed that more than $90 \%$ of dLGN-INs are born between E10.5-E13.5 with a peak of neurogenesis at days E11.5 and E12.5 combined (38.7 \pm 0.3 and $37.7 \pm 5.2$, respectively; \%, mean \pm standard error; $n=3$ brains per time point) (Supplementary Fig. 1). We then introduced a plasmid driving constitutive enhanced green fluorescent protein (eGFP) expression in the proliferative zone of the dorsal midbrain at E12.5, via in utero electroporation and analysed the electroporated brains at P3 (Supplementary Fig. 1). Electroporated brains showed extensive eGFP labelling across the tectum with some eGFP expression retained in the glia cells that form the ependymal layer lining the midbrain aqueduct, but not those lining the diencephalic third ventricle (Supplementary Fig. 1). The restricted labelling of midbrain ependymal cells at P3 was taken as a consequence of the specific targeting of dorsal midbrain progenitors at E12.5. Importantly, eGFP ${ }^{+}$cells were also detected in the thalamus (Supplementary Fig. 1), predominantly in the dLGN (50\%), but also in the vLGN/IGL complex (11\%) and in the remainder of the thalamus (39\%) - principally in the lateral posterior and ventrobasal thalamic nuclei. Immunodetection of GABA confirmed the inhibitory identity of the dLGN GFP ${ }^{+}$cells (Supplementary Fig. 1).

All evidence so far weights strongly in favour of a tectal origin for all dLGN-INs. Yet, our in utero dorsal midbrain labelling of progenitors and precursors cannot categorically exclude the possibility of unintentional labelling in the nearby diencephalic pretectal $(\mathrm{p} 1)$ territory.

To exclude the pretectum as a dLGN-IN-contributing territory, we took advantage of the known requirement for Gata2 in inhibitory fate identity and used the $E n 1^{\text {cre/ }+}$; Gata $2^{f l x / f l x}$ conditional mutant to specifically ablate the GABAergic fate in the dorsal midbrain while preserving Gata2 expression and inhibitory neurogenesis in all diencephalic compartments, including the pretectum $(\mathrm{p} 1, \mathrm{p} 2 \text { and } \mathrm{p} 3)^{48}$. Gata2 is required for Sox14 expression within Ascl1 ${ }^{+}$inhibitory lineages across the midbrain and diencephalic p1, p2 and p3, and is transiently coexpressed with Sox 14 on cell cycle exit (Fig. $7 \mathrm{f})^{19}$ (K. Achim, A. Kirjavainen and J.P., unpublished). Remarkably, the dLGN of $\mathrm{En}^{\text {crel }+}$; Gata2 $2^{f x / f l x}$ shows a $91 \%$ reduction of GABAergic cells

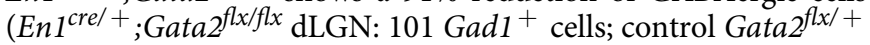
dLGN: $1,090 \mathrm{Gad}^{+}{ }^{+}$cells. On the basis of 25 and 26 sections, respectively, covering the entire rostro-caudal span of the nucleus. $N=2$ brains per genotype.), which correlates with the similarly strong reduction of inhibitory neurons in the dorsal midbrain (Fig. 7g). In contrast, other GABA-rich diencephalic territories, as well as the ventral midbrain, are grossly unaffected (Fig. $7 \mathrm{~g}$ ). Hence a conditional mutagenesis strategy that disrupts the acquisition of the inhibitory fate in the midbrain but not in the diencephalon, confirms that the tectum and not the nearby pretectum, is the main source of dLGN-INs.

\section{Discussion}

We provide the first experimental evidence for a tectal origin of thalamic interneurons and redefined dLGN-INs as an Nkx2.2$\mathrm{Gata}_{2}{ }^{+} \mathrm{Otx}_{2}{ }^{+}$Sox $14^{+}$lineage, requiring Gata2, Otx2 and Sox14 expression. Using optogenetics, we exploit this previously unappreciated genetic signature to demonstrate a frequencydependent switch from a phasic to a tonic form of inhibition within the visual thalamus.

Ventro-dorsal oriented tangential migration is known to underlie the positioning of interneurons into the pre-existing excitatory pyramidal neuron organization of the neocortex ${ }^{3}$, and a similar developmental scenario is considered to take place within the thalamus with interneurons migrating from the ventral (p3) region of the thalamus ${ }^{26}$. In support of this established textbook view ${ }^{27}$, tangentially migrating GABAergic precursors that move in a rostro-caudal direction in the developing thalamus have been described ${ }^{49,50}$, although the fate of these neurons does not appear to be local thalamic interneurons, but rather to coincide with an early wave of migrating $\mathrm{pTh}-\mathrm{R}$ precursors, which peaks at E12.5-E13.5 and is also labelled by Sox14 expression ${ }^{17}$. This early wave of tangentially migrating Sox $14^{+}$ neurons contributes inhibitory cells to the perilateral habenula and the nucleus posterior limitans, at the dorsal and caudal edges of the thalamus, respectively ${ }^{17}$. The bulk of the GABAergic neurogenesis that takes place in the pTh- $\mathrm{R}$ will contribute to the IGL and part of the $\mathrm{VLGN}^{15,17,19,22}$. It has been observed that thalamic interneurons appear within the thalamus after birth ${ }^{25,26}$ We now show that none of the diencephalic progenitor domains (p1, p2 and p3) is a likely source of dLGN-INs. Our observation that dLGN-INs are an immigrant tectal population reinforces the important role of tangential migration ${ }^{51}$ in seeding distant networks with GABAergic interneurons. In contrast to cortical interneurons, which arise and migrate within the boundaries of the telencephalon, we suggest that thalamic interneurons are not diencephalic, but born in an evolutionary and developmentally distinct brain region: the midbrain. Furthermore, the genetic programme leading to tangential migration must also be different, as Arx, a key regulator of tangential migration in cortical interneurons ${ }^{52}$, is not expressed by Sox $14^{+}$neurons.

Tangential migration is likely controlled by genetically encoded programmes of the type described in this paper, but maturation of the visual circuitry in the dLGN also requires presynaptic activity in the retinogeniculate pathway ${ }^{53-57}$. Recently, spontaneous retinal waves have been shown to influence the final positioning of dLGN-INs along the medio-lateral axis of the nucleus $^{25}$. During this critical time window, postsynaptic activity is also likely to play an important role ${ }^{58,59}$ and GABAergic interneurons may contribute to the functional maturation of visual circuits ${ }^{60,61}$. Although we did not report any gross abnormality in the segregation of retinal afferents in the dLGN of Sox14 knockout mice, more detailed future analysis is required to establish a role for dLGN-INs in visual circuit refinement.

Unpublished data from our laboratory suggest that other interneuron populations in the ventrobasal and lateroposterior thalamic nuclei are also strongly reduced in the Gata $2^{f x} f f x ; E n 1^{\text {cre }}$ and Sox14 knockout brains and are likely of mesencephalic origin. The relative abundance of inhibitory interneurons across various thalamic nuclei of different mammalian species may correlate with the relative complexity of thalamic circuits ${ }^{62}$. Future comparative work is required to establish whether the tectal origin of thalamic inhibitory interneurons is shared beyond the visual thalamus in carnivorans and primates.

Nevertheless, by exploiting the genetic signature of dLGN-INs we were able to demonstrate, using an optogenetic strategy, that Sox $14^{+}$dLGN-INs generate both phasic and tonic forms of inhibition, but activation of extrasynaptic $\delta$ - $\mathrm{GABA}_{\mathrm{A}} \mathrm{Rs}$ was enhanced when dLGN-IN firing rates were high. Indeed, simultaneous paired recording between dLGN-INs and thalamic relay neurons was characterized by a dominance of extrasynaptic $\delta$-GABA ${ }_{A} R$ activation. The low prevalence of AP-evoked IPSCS in these simultaneous recordings may, in part, be explained by the synaptic fatigue that is clearly observed with optogenetic stimulation of GABA release from dLGN-IN terminals. A previous study ${ }^{41}$ has demonstrated that evoked IPSCs in the ventrobasal thalamus also contained a slow component that was mediated by extrasynaptic $\delta$-GABA $\mathrm{A}$ Rs. Other studies have 
shown that genetic removal of synaptic $G_{A B A}$ Rs from the ventrobasal thalamus did not disturb thalamocortical slow oscillations and sleep spindles ${ }^{63}$. The fact that dLGN-INs are capable of activating extrasynaptic $\delta$-GABA ${ }_{A}$ Rs may explain why thalamocortical oscillations can be maintained in the absence of synaptic $\mathrm{GABA}_{\mathrm{A}}$ receptors.

Tonic inhibition of the type we describe in this study will be driven by visual input to the dLGN, whereas in other regions of sensory thalamus the GABAergic drive will be generated solely from the surrounding RTN. The identity of the retinal ganglion cell (RGC) types that make synaptic connections with Sox $14^{+}$ dLGN-INs will need to be established, but an intriguing scenario would see them innervated by luminance-detecting intrinsically photosensitive RGCs $^{64}$ providing a potential mechanism to regulate excitability of relay neurons to environmental luminance. The tools we have developed for genetically targeting Sox $14^{+}$dLGN-INs will help us identify the RGC types that excite thalamic interneurons.

\section{Methods}

Animals. The Sox14::Cre-targeting vector was constructed by replacing the Sox14coding sequence with a cassette consisting of a $5^{\prime}$ splice substrate followed by the cre gene and a bovine growth hormone polyadenylation signal (bGHpA). A neomycin gene cassette was cloned downstream of the $b G H p A$ signal for positive selection in embryonic stem cells. The Sox $14::$ Cre targeting vector was electroporated into mouse embryonic stem cells $(129 \mathrm{sv} / \mathrm{ev})$, selected with G418 and homologous recombinants were identified by Southern blot analysis. The targeted embryonic stem cells were injected into blastocysts, and chimeras were crossed to C57BL/6J mice. The PGKneo cassette was removed after crossing with the FLPeR mice ${ }^{65}$. The Sox $14^{G f p /+}$ (refs 17,66) and Sox $14^{\text {cre } /+}$ mouse lines were maintained in the C57Bl/6 background in the animal facilities of King's College London and housed under standard conditions. All experimental procedures described have received internal approval by the King's College London Ethical Committee and are covered by a UK Home Office Licence. The genetic background and age of mouse lines used are described in the relevant 'Results' and 'Methods' sections. Animals of both sexes were used to produce the data presented.

Stereotaxic brain injections. For optogenetics, Sox14 $4^{\text {cre }}+\mathrm{P} 16$ pups were anaesthetized with isoflurane and full anaesthesia maintained throughout the procedure. The head was fixed in a small animal stereotaxic frame (World Precision Instruments), the skull exposed via a $1 \mathrm{~cm}$ longitudinal skin incision and the initial dLGN coordinates defined as $y: 1.7 ; x: 2.4 ; z: 3.0$ from lambda, then optimized for each litter. Sox $14^{\text {cre } /+}$ mice were injected bilaterally using borosilicate glass needles connected to an air injector system (Narishige). With an approximate volume of $0.2-0.3 \mu \mathrm{l}$ of $r A A V 2-E F 1 a-D I O-h C h R 2(H 134 R)-E Y F P-$ WPRE (Addgene plasmid 20298). Electrophysiological experiments were performed 2 weeks after surgery.

BrdU labelling and detection. Wild-type C57BL/6 dams were mated with heterozygous Sox $14^{\mathrm{Gfp} /+}$ males. BrdU (Sigma, B5002) was administered intraperitoneally to pregnant mice $\left(0.2 \mathrm{mg} \mathrm{g}^{-1}\right)$ at 09:00 between E10.5 and 13.5, estimated from the occurrence of a vaginal plug (morning of the day the plug was detected was designated E0.5). Gfp heterozygous pups were perfused at P7 with $4 \%$ paraformaldehyde (PFA) and treated for cryosectioning as above. For BrdU/Sox14 double labelling, $40 \mu \mathrm{m}$ floating sections were cut, followed by denaturation with $1 \mathrm{M} \mathrm{HCl}$ in $\mathrm{H} 2 \mathrm{O}$ at $45^{\circ} \mathrm{C}$ for $30 \mathrm{~min}$ and neutralization with three washes of phosphate-buffered saline (PBS; $\mathrm{pH} 7.4$ ) for $10 \mathrm{~min}$. Sections were then blocked in $2 \%$ normal goat serum (NGS), $0.3 \%$ Triton-X, in PBS for $1 \mathrm{~h}$ at room temperature, and probed with rat antiBrdU (1:200, in $2 \%$ NGS, $0.3 \%$ Triton-X, in PBS, OBT0030CX Bio-Rad) and chicken anti-GFP (Ab13970 Abcam) primary antibodies at $4{ }^{\circ} \mathrm{C}$ for $48 \mathrm{~h}$, followed by fluorescent Goat Alexa-568 anti-rat and Alexa488 anti-chicken (A11039, Invitrogen) secondary antibodies. Counting of BrdU ${ }^{+}$ and Gfp ${ }^{+}$double-positive cells in the Sox $14^{G f p}++$ dLGN used every second section, covering the whole span of the dLGN ( $n=3$ brains per developmental stage).

In utero brain injections and electroporation. To maximize litter size and the chances of survival of the litter, we crossed Sox $14^{\text {cre } /+}$ males to CD1 dams. The CD1 pregnant dam was maintained under full anaesthesia with isoflurane throughout the procedure. Incision was made through the skin and the abdominal wall to expose the uterine horns. An approximate volume of $0.2-5 \mu \mathrm{l}$ of $r A A V 2$ EF1a-DIO-tdTomato-WPRE produced as serotype 1 was injected using borosilicate glass needles connected to an air injector system (Narishige). A stereomicroscope was used to target the injection to the embryonic tectum of E15.5 embryos. Mice were killed at P14 and genotyped for presence of the cre allele. The brains were sectioned at $60-80 \mu \mathrm{m}$ and images were acquired with a spinning disk confocal microscope (Eclipse Ni-E Upright, Nikon). Three Sox $14^{\text {cre } /+}$ embryos were injected in the tectum, and on postnatal analysis displayed selective enrichment of tdTomato-labelled cells in the LGN. For control three Sox $14^{\text {cre } /+}$ embryos were injected in the hypothalamus. On postnatal analysis these showed labelling of hypothalamic Sox $14^{+}$neurons, but not the thalamus. Counting of tdTomatoexpressing cells in the LGN was done on every second section, covering the whole span of the LGN (145 cells from 2 brains). Focal labelling of cre-expressing cells was consistently observed ipsilateral to the injection site, which excludes the possibility that labelling occurs via accidental leakage of the virus in the brain ventricles. For in utero electroporations, reporter and Cre-dependent plasmids

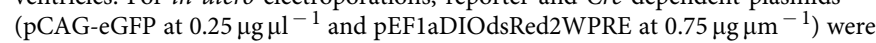
injected in the midbrain ventricle at E12.5. Five voltage pulses $(37 \mathrm{mV}, 50 \mathrm{~ms})$ were then applied using Electroporator CUY21 (Nepa Gene) and tweezers with asymmetric round plate platinum electrodes (1-5 mm diameter; CUY650P1-5 Nepa Gene) to allow for more focal targeting. The brains were collected at P3 and processed and analysed as above ( $n=2$ brains).

Anterograde tracing of the optic tract. P19 Sox $14^{G f p /+}$ mice were anaesthetized with isoflurane and a small incision was performed through the cornea of one eye to facilitate access to the intravitreum. The eye cavity was filled with Alexa594-conjugated CTb at $1 \mu \mathrm{gll}^{-1}$ in PBS (Life Technologies) using a pulled borosilicate glass needle and an injector pump (Picospritzer III). Three days later animals were perfused with $4 \%$ PFA/PBS under terminal anaesthesia. Brains were postfixed in $4 \%$ PFA solution at $4{ }^{\circ} \mathrm{C}$ overnight and further processed for cryosectioning and immunohistochemistry (IHC) to amplify the Gfp signal.

IHC and RNA in situ hybridization. Mice were transcardially perfused with $4 \%$ PFA in PBS and the brains postfixed at $4{ }^{\circ} \mathrm{C}$ overnight for IHC (longer for in situ hybridization (ISH)). Brains were equilibrated in $30 \%$ sucrose/PBS, embedded in OCT freezing compound and cut on a cryotome at $80-100 \mu \mathrm{m}$ for IHC, or $20 \mu \mathrm{m}$ for ISH and IHC on embryonic tissue. IHC was performed on floating sections using $7 \%$ goat serum/PBS with $0.3 \%$ TritonX-100 as blocking and antibodybinding solution. The following primary antibodies were incubated on sections overnight at $4{ }^{\circ} \mathrm{C}$ : rabbit aOtx1-2 (1:1,200, ab21990 Abcam); chick aGfp (1:10,000, ab13970 Abcam); rabbit aCasp3 (1:300, ab13847 Abcam); rabbit adsRed (1:200 Clontech); mouse aTUJ1 (1:300, MMS-435 Covance); rabbit aGata2 (1:200, H-116 Santa Cruz); mouse aMash1 (Ascl1) (1:100, 556604 BD Biosciences); mouse aNkx2.2 (1:50, 74.5A5 DSHB); and rabbit aGABA (1:2,000, A2052 Sigma). All secondary antibodies were Alexa-conjugated goat aIgG (1:500, Life Technologies). ISH was performed as previously described ${ }^{17}$. The Otx2 antisense RNA probe was transcribed in vitro from a full-length cDNA template (IMAGE: 4527414). The Gad1 probe was a kind gift from John Rubenstein, UCSF, San Francisco, USA. On satisfactory colour reaction with Fast Red substrate, sections were immunoreacted with a chick antiGfp antibody for $48 \mathrm{~h}$ at $4{ }^{\circ} \mathrm{C}$, and fluorescent images acquired using a spinning disk confocal microscope (Eclipse Ni-E Upright, Nikon). Counting of Sox $14^{+}$and Otx2 ${ }^{+}$cells in the Sox14 Gfp/+ LGN at P3 was done on every fifth section $(20 \mu \mathrm{m}$ thickness), covering the whole span of the LGN

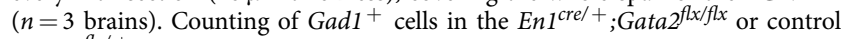
$\left(\right.$ Gata $\left.2^{f l x /+}\right)$ dLGN at P7 was done in the same way ( $n=2$ brains per genotype) Counting of $\mathrm{GABA}^{+}{ }^{\mathrm{tdT}}$ Tomato ${ }^{+}$cells in the $N k \times 2.2^{\text {cre/ }+} ;$ R26-LSL-tdTomato $\mathrm{dLGN}$ at P30 used every second section ( $60 \mu \mathrm{m}$ thickness), covering the whole extent of the dLGN ( $n=3$ brains).

Ex vivo time-lapse imaging. Brains of $\mathrm{P} 0.5$ Sox $14^{\mathrm{Gfp} /+}$ mice were dissected on ice and embedded in $4 \%$ low-melting-temperature agarose. The $250 \mu \mathrm{m}$-thick sections were cut with vibratome (Leica VT 1200S) in ice-cold sucrose solution (all in mM): 70 sucrose; $86 \mathrm{NaCl} ; 4 \mathrm{KCl} ; 1 \mathrm{NaH}_{2} \mathrm{PO}_{4} ; 1.4 \mathrm{MgCl}_{2} ; 26 \mathrm{NaHCO}_{3} ; 1 \mathrm{CaCl}_{2}$; and 24 glucose, and held in ice-cold Krebs buffer post sectioning (all in mM): $12.6 \mathrm{NaCl}$; $0.25 \mathrm{KCl} ; 0.12 \mathrm{NaH}_{2} \mathrm{PO}_{4} ; 0.12 \mathrm{MgCl}_{2} ; 0.21 \mathrm{CaCl}_{2} ; 25 \mathrm{NaHCO}_{3} ; 11$ glucose, and supplemented with HEPES (Invitrogen), penicillin, streptomycin and gentamicin (Invitrogen). Sections containing dLGN were placed on Millicell culture filters (Millipore, $0.4 \mu \mathrm{m}, 30 \mathrm{~mm}$ diameter), floating on pre-warmed MEM medium (Gibco) supplemented with glucose, FCS, penicillin and streptomycin (Invitrogen). After $1 \mathrm{~h}$ the medium was replaced with Neurobasal (Gibco), supplemented with B27 (Gibco), glutamine (Invitrogen), glucose, penicillin and streptomycin (Invitrogen). After $16 \mathrm{~h}$ in culture the sections were imaged with an inverted laser scanning spectral confocal microscope (Leica TCS SP2 RS) using water-immersion $\times 25$ objective, and maintained at $37^{\circ} \mathrm{C}$ with $95 \% \mathrm{O}_{2} / 5 \% \mathrm{CO}_{2}$. Images were taken every 60 min over a $26 \mathrm{~h}$ period and analysed for cell movement in FIJI using the Pairwise stitching and Manual Tracking plugins.

Quantification of migrating neurons. For each Sox $14^{G f p /+}$ brain (P0-P2), $z$-stack images of the dLGN with interplanar separation of $1.8 \mu \mathrm{m}$, spanning on average $50 \mu \mathrm{m}$, were acquired with a spinning disk confocal microscope (Eclipse $\mathrm{Ni}$-E Upright, Nikon), taken at $\times 20$ magnification. Every second dLGN-containing section was imaged for each brain (3-4 sections per brain). The acquired $z$-stacks were analysed for the migratory morphology of dLGN Sox $14^{+}$cells by marking the orientation of their leading processes using the Icy software arrow 
tool. Leading processes were identifiable in 165 out of 445 (37\%) Gfp ${ }^{+}$dLGN cells. To quantify their orientation, the angles of the arrows were determined in FIJI relative to the $x$ axis of the dLGN (width). The latter was defined by taking the $x$ axis as parallel to the base of the nucleus and positive in the medial direction, and the $y$ axis as the midline perpendicular to the base and positive in the dorsal direction. The angles were plotted on a histogram, using $20^{\circ}$ bins. In addition, the component of the orientation along the dorso-ventral and latero-medial directions for each leading process was determined by taking the sine and cosine of the angles, respectively. This produces indices between 1 and -1 for each direction. In the latero-medial axis, an index of 1 corresponds to purely medial orientation, and of -1 to purely lateral orientation. In the dorso-ventral direction, an index of 1 corresponds to purely dorsal orientation, and -1 to purely ventral orientation. The dorso-ventral and latero-medial indices for each leading process were plotted on a scatter plot in the dLGN $x-y$ coordinate system across all sections, for each developmental stage, using Microsoft Excel. The average value of each index is also shown for each plot, where an average of 0 would suggest equal representation of all orientations. As the indices were not normally distributed, the Wilcoxon signed rank test was used to establish that average indices were significantly different from 0 .

Three-dimensional-image reconstruction of the dLGN. Z-stack images, with an interplanar separation of $11 \mu \mathrm{m}$, of the sox $14 \mathrm{Gfp} /+\mathrm{P} 3 \mathrm{dLGN}$ were acquired with a spinning disk confocal microscope (Eclipse Ni-E Upright, Nikon), using a $\times 10$ objective. Every dLGN-containing section was imaged and the acquired $z$-stacks were aligned and assembled in rostral to caudal progression in FIJI using the TrakEM2 plugin.

Acute slice preparation and whole-cell patch clamp recording. Brain slices were obtained from both male and female mice at postnatal 21-30 days, which were killed by cervical dislocation followed by decapitation. The brain was rapidly removed from the skull and immersed in ice-cold slicing solution composed of (in mM) the following: $85 \mathrm{NaCl} ; 2.5 \mathrm{KCl} ; 1 \mathrm{CaCl}_{2} ; 4 \mathrm{MgCl}_{2} ; 1.25 \mathrm{NaH}_{2} \mathrm{PO}_{4} ; 26$ $\mathrm{NaHCO}_{3} ; 75$ sucrose; and 25 glucose, $\mathrm{pH}$ 7.4, when bubbled with $95 \% \mathrm{O}_{2}$ and $5 \%$ $\mathrm{CO}_{2}$. Coronal brain slices $(250 \mu \mathrm{m}$ thickness) were cut (Campden Instruments) and immediately transferred to a holding chamber containing slicing artifical cerebrospinal fluid (ACSF) aerated with $95 \% \mathrm{O}_{2} / 5 \% \mathrm{CO}_{2}$. Slices were then transferred to a $37^{\circ} \mathrm{C}$ heat block for $10 \mathrm{~min}$, after which the slicing ACSF was exchanged for recording ACSF (in mM: $\mathrm{NaCl} 125 ; \mathrm{KCl} 2.5 ; \mathrm{CaCl}_{2} 2 ; \mathrm{MgCl}_{2} 1 ; \mathrm{NaH}_{2} \mathrm{PO}_{4} 1.25$; $\mathrm{NaHCO}_{3}$ 26; and glucose 11, pH 7.4, when bubbled with $95 \% \mathrm{O}_{2}$ and $5 \% \mathrm{CO}_{2}$ ). The slices were incubated in the recording ACSF at room temperature for at least another $30 \mathrm{~min}$ before electrophysiological recordings. The recording chamber was perfused with carbogen-saturated ACSF with a flow rate of $2-5 \mathrm{ml} \mathrm{min}^{-1}$ using a gravity perfusion system. Patch pipettes were fabricated with a two-step vertical puller (Narishige, PC-10), with tip resistance at $4-8 \mathrm{M} \Omega$ when back-filled with internal solution. For whole-cell voltage-clamp recordings, the patch pipettes were filled with Cs-based internal solution containing following (in $\mathrm{mM}$ ): $\mathrm{CsCl} 140$; $\mathrm{NaCl} 4, \mathrm{CaCl}_{2}$ 0.5; HEPES 10; EGTA 5; and Mg-ATP 2; $\mathrm{pH}$ 7.3, adjusted with $\mathrm{CsOH}$. For whole-cell current-clamp recordings, we used internal solution containing the following (in $\mathrm{mM}$ ): $145 \mathrm{~K}$-gluconate; $4 \mathrm{NaCl} ; 5 \mathrm{KCl} ; 0.5 \mathrm{CaCl}_{2} ; 5 \mathrm{EGTA}$; 10 HEPES; $4 \mathrm{Mg}$-ATP; and 5 sucrose, $\mathrm{pH} 7.3$, adjusted with KOH. Fixed-stage upright microscope (Olympus BX51W1) fitted with a water-immersion objective (Olympus, $\times 60$ ) and a non-immersion objective (Zeiss, $\times 1.25)$ was used to visualize the neurons in slices. Whole-cell recordings were performed with a Multiclamp 700B amplifier (Molecular Devices) at room temperature. The analogue output was low-pass filtered at $10 \mathrm{kHz}$, digitized at $20 \mathrm{kHz}$ using a BNC-2120 device (National Instruments), and analysed using WINWCP and WINEDR software (John Dempster, University of Strathclyde, UK). In most cases, biocytin $\left(2 \mathrm{mg} \mathrm{ml}^{-1}\right)$ was added to the internal solution and patch pipette was slowly pulled away from the neuron after whole-cell recording. The slice tissue was then preserved in $4 \%$ PFA for over $48 \mathrm{~h}$. To start the immunostaining process, PFA was washed off with ice cold PBS three times, 10 min each time. Slices were then blocked and permeabilized with 5\% donkey serum and $0.2 \%$ TritonX-100 in PBSbased solution at room temperature for 1-2 h. After washing with PBS for $10 \mathrm{~min}$, slices were submerged in $2 \mathrm{mg} \mathrm{ml}^{-1}$ streptavidin, Alexa Fluor 555 Conjugate (Life Technologies)-added PBS solution with $1 \%$ donkey serum and $0.2 \%$ Triton X-100 for $3-4 \mathrm{~h}$ at room temperature. Subsequently, slices were washed again in PBS for three times, 10 min each, and then mounted on slides with mounting medium (H1000 , Vectashield).

Optogenetic stimulation of Sox14 ${ }^{+}$dLGN-INs. A blue $(470 \mathrm{~nm})$ collimated LED (M470L3-C1, Thorlabs) was mounted to the back of the microscope, and focused through the objective lens. The optical power emitted by our $\times 63$ waterimmersion lens increased linearly to a maximum power of $70 \mu \mathrm{W} \mathrm{mm} m^{-2}$ at $1 \mathrm{~V}$. A $1 \mathrm{~ms}, 1 \mathrm{~V}$ brief pulse protocol gave rise to a transient response that peaked at $40 \mu \mathrm{W} \mathrm{mm}{ }^{-2}$ with a $10-90 \%$ rise time of $0.73 \mathrm{~ms}$ and a decay constant of $9.65 \mathrm{~ms}$; as determined from a single exponential fit. In a series of calibration experiments we observed that CHR2-evoked responses were not detected when the illumination spot was moved $\sim 200 \mu \mathrm{m}$ from the recorded cell, corresponding to an illumination area of $0.03 \mathrm{~mm}^{2}$.
Quantification of tonic and phasic conductance changes. All-point histograms were constructed from $1 \mathrm{~s}$ epochs of the holding current recorded under conditions, where the tonic conductance was not present before chloride loading, present in control conditions, blocked using $\mathrm{GABA}_{\mathrm{A}}$ receptor antagonists or enhanced by the allosteric modulator DS2. A single Gaussian function was used to estimate the mean holding current and the tonic conductance was calculated from the driving force according to the relationship; $G_{\text {tonic }}=$ change in mean holding current driving force. The quality of a linear regression was established using the coefficient of determination or $R^{2}$, with values closer to 1 indicating a high degree of confidence in the regression model. All statistical hypothesis testing was performed assuming normal data distributions unless otherwise stated.

Data availability. The data that support the findings of this study are available on request from the corresponding authors (S.G.B. and A.D.).

\section{References}

1. Le Magueresse, C. \& Monyer, H. GABAergic interneurons shape the functional maturation of the cortex. Neuron 77, 388-405 (2013).

2. Akerman, C. J. \& Cline, H. T. Refining the roles of GABAergic signaling during neural circuit formation. Trends Neurosci. 30, 382-389 (2007).

3. Marin, O. \& Muller, U. Lineage origins of GABAergic versus glutamatergic neurons in the neocortex. Curr. Opin. Neurobiol. 26, 132-141 (2014).

4. Marin, O. \& Rubenstein, J. L. Cell migration in the forebrain. Annu. Rev. Neurosci. 26, 441-483 (2003).

5. Huang, Z. Molecular regulation of neuronal migration during neocortical development. Mol. Cell. Neurosci. 42, 11-22 (2009).

6. Petilla Interneuron Nomenclature, G. et al. Petilla terminology: nomenclature of features of GABAergic interneurons of the cerebral cortex. Nat. Rev. Neurosci. 9, 557-568 (2008).

7. DeFelipe, J. et al. New insights into the classification and nomenclature of cortical GABAergic interneurons. Nat. Rev. Neurosci. 14, 202-216 (2013).

8. Fishell, G. \& Rudy, B. Mechanisms of inhibition within the telencephalon: 'where the wild things are'. Annu. Rev. Neurosci. 34, 535-567 (2011).

9. Houser, C. R., Vaughn, J. E., Barber, R. P. \& Roberts, E. GABA neurons are the major cell type of the nucleus reticularis thalami. Brain Res. 200, 341-354 (1980).

10. Hunt, C. A., Pang, D. Z. \& Jones, E. G. Distribution and density of GABA cells in intralaminar and adjacent nuclei of monkey thalamus. Neuroscience $\mathbf{4 3}$, 185-196 (1991).

11. Puelles, L. \& Rubenstein, J. L. Forebrain gene expression domains and the evolving prosomeric model. Trends Neurosci. 26, 469-476 (2003).

12. Thompson, C. L. et al. A high-resolution spatiotemporal atlas of gene expression of the developing mouse brain. Neuron 83, 309-323 (2014).

13. Altman, J. \& Bayer, S. A. Development of the rat thalamus: III. Time and site of origin and settling pattern of neurons of the reticular nucleus. J. Comp. Neurol. 275, 406-428 (1988).

14. Altman, J. \& Bayer, S. A. Development of the rat thalamus: VI. The posterior lobule of the thalamic neuroepithelium and the time and site of origin and settling pattern of neurons of the lateral geniculate and lateral posterior nuclei. J. Comp. Neurol. 284, 581-601 (1989).

15. Vue, T. Y. et al. Characterization of progenitor domains in the developing mouse thalamus. J. Comp. Neurol. 505, 73-91 (2007)

16. Song, H. et al. Ascl1 and Helt act combinatorially to specify thalamic neuronal identity by repressing Dlxs activation. Dev. Biol. 398, 280-291 (2015).

17. Delogu, A. et al. Subcortical visual shell nuclei targeted by ipRGCs develop from a Sox14+-GABAergic progenitor and require Sox14 to Regulate daily activity rhythms. Neuron 75, 648-662 (2012).

18. Sellers, K., Zyka, V., Lumsden, A. G. \& Delogu, A. Transcriptional control of GABAergic neuronal subtype identity in the thalamus. Neural Dev. 9, 14 (2014).

19. Virolainen, S. M., Achim, K., Peltopuro, P., Salminen, M. \& Partanen, J. Transcriptional regulatory mechanisms underlying the GABAergic neuron fate in different diencephalic prosomeres. Development 139, 3795-3805 (2012).

20. Cobos, I., Broccoli, V. \& Rubenstein, J. L. The vertebrate ortholog of Aristaless is regulated by Dlx genes in the developing forebrain. J. Comp. Neurol. 483, 292-303 (2005).

21. Stühmer, T., Anderson, S. A., Ekker, M. \& Rubenstein, J. L. R. Ectopic expression of the Dlx genes induces glutamic acid decarboxylase and Dlx expression. Development 129, 245-252 (2002).

22. Jeong, Y. et al. Spatial and temporal requirements for sonic hedgehog in the regulation of thalamic interneuron identity. Development 138, 531-541 (2011).

23. Kataoka, A. \& Shimogori, T. Fgf8 controls regional identity in the developing thalamus. Development 135, 2873-2881 (2008).

24. Scholpp, S. et al. Her6 regulates the neurogenetic gradient and neuronal identity in the thalamus. Proc. Natl Acad. Sci. USA 106, 19895-19900 (2009).

25. Golding, B. et al. Retinal input directs the recruitment of inhibitory interneurons into thalamic visual circuits. Neuron 81, 1057-1069 (2014). 
26. Jones, E. G. Dichronous appearance and unusual origins of GABA neurons during development of the mammalian thalamus. Thalamus Relat. Syst. 1, 283-288 (2002).

27. Jones, E. G. in The Thalamus 2nd edn, Vol. I, 533 (Cambridge Univ. Press, 2007).

28. Benson, D. L., Isackson, P. J., Gall, C. M. \& Jones, E. G. Contrasting patterns in the localization of glutamic acid decarboxylase and $\mathrm{Ca} 2+/$ calmodulin protein kinase gene expression in the rat central nervous system. Neuroscience 46, 825-849 (1992).

29. Singer, W. \& Bedworth, N. Inhibitory interaction between X and Y units in the cat lateral geniculate nucleus. Brain Res. 49, 291-307 (1973).

30. Sillito, A. M. \& Kemp, J. A. The influence of GABAergic inhibitory processes on the receptive field structure of $\mathrm{X}$ and $\mathrm{Y}$ cells in cat dorsal lateral geniculate nucleus (dLGN). Brain Res. 277, 63-77 (1983).

31. Williams, S. R., Turner, J. P., Anderson, C. M. \& Crunelli, V. Electrophysiological and morphological properties of interneurones in the rat dorsal lateral geniculate nucleus in vitro. J. Physiol. 490, 129-147 (1996).

32. Dubin, M. W. \& Cleland, B. G. Organization of visual inputs to interneurons of lateral geniculate nucleus of the cat. J. Neurophysiol. 40, 410-427 (1977).

33. Ralston, 3rd H. J. Evidence for presynaptic dendrites and a proposal for their mechanism of action. Nature 230, 585-587 (1971).

34. Sherman, S. M. Interneurons and triadic circuitry of the thalamus. Trends Neurosci. 27, 670-675 (2004).

35. Errington, A. C., Di Giovanni, G., Crunelli, V. \& Cope, D. W. mGluR control of interneuron output regulates feedforward tonic GABAA inhibition in the visual thalamus. J. Neurosci. 31, 8669-8680 (2011).

36. McAlonan, K., Cavanaugh, J. \& Wurtz, R. H. Guarding the gateway to cortex with attention in visual thalamus. Nature 456, 391-394 (2008).

37. Steriade, M. Sleep, epilepsy and thalamic reticular inhibitory neurons. Trends Neurosci. 28, 317-324 (2005).

38. Acuna-Goycolea, C., Brenowitz, S. D. \& Regehr, W. G. Active dendritic conductances dynamically regulate GABA release from thalamic Interneurons. Neuron 57, 420-431 (2008)

39. Casale, A. E. \& McCormick, D. A. Active action potential propagation but not initiation in thalamic interneuron dendrites. J. Neurosci. 31, 18289-18302 (2011).

40. Govindaiah, G. \& Cox, C. L. Modulation of thalamic neuron excitability by orexins. Neuropharmacology 51, 414-425 (2006).

41. Herd, M. B., Brown, A. R., Lambert, J. J. \& Belelli, D. Extrasynaptic GABA(A) receptors couple presynaptic activity to postsynaptic inhibition in the somatosensory thalamus. J. Neurosci. 33, 14850-14868 (2013).

42. Bright, D. P., Aller, M. I. \& Brickley, S. G. Synaptic release generates a tonic $\mathrm{GABA}(\mathrm{A})$ receptor-mediated conductance that modulates burst precision in thalamic relay neurons. J. Neurosci. 27, 2560-2569 (2007).

43. Jensen, M. L. et al. A study of subunit selectivity, mechanism and site of action of the delta selective compound 2 (DS2) at human recombinant and rodent native GABA(A) receptors. Br. J. Pharmacol. 168, 1118-1132 (2013).

44. Ye, Z., McGee, T. P., Houston, C. M. \& Brickley, S. G. The contribution of delta subunit-containing GABAA receptors to phasic and tonic conductance changes in cerebellum, thalamus and neocortex. Front. Neural Circuits 7, 203 (2013).

45. Wafford, K. A. et al. Novel compounds selectively enhance delta subunit containing GABA A receptors and increase tonic currents in thalamus. Neuropharmacology 56, 182-189 (2009).

46. Balderes, D. A., Magnuson, M. A. \& Sussel, L. Nkx2.2:Cre knock-in mouse line: a novel tool for pancreas- and CNS-specific gene deletion. Genesis 51, 844-851 (2013).

47. Qi, Y. et al. Control of oligodendrocyte differentiation by the Nkx2.2 homeodomain transcription factor. Development 128, 2723-2733 (2001).

48. Kala, K. et al. Gata2 is a tissue-specific post-mitotic selector gene for midbrain GABAergic neurons. Development 136, 253-262 (2009).

49. Ortino, B., Inverardi, F., Morante-Oria, J., Fairén, A. \& Frassoni, C. Substrates and routes of migration of early generated neurons in the developing rat thalamus. Eur. J. Neurosci. 18, 323-332 (2003).

50. Puelles, L., Sanchez, M. P., Spreafico, R. \& Fairen, A. Prenatal development of calbindin immunoreactivity in the dorsal thalamus of the rat. Neuroscience 46, 135-147 (1992).

51. Marin, O. Cellular and molecular mechanisms controlling the migration of neocortical interneurons. Eur. J. Neurosci. 38, 2019-2029 (2013).

52. Colasante, G. et al. Arx is a direct target of Dlx2 and thereby contributes to the tangential migration of GABAergic interneurons. J. Neurosci. 28, 10674-10686 (2008).

53. Stellwagen, D. \& Shatz, C. J. An instructive role for retinal waves in the development of retinogeniculate connectivity. Neuron 33, 357-367 (2002).
54. Hooks, B. M. \& Chen, C. Distinct roles for spontaneous and visual activity in remodeling of the retinogeniculate synapse. Neuron 52, 281-291 (2006).

55. Chen, C. \& Regehr, W. G. Developmental remodeling of the retinogeniculate synapse. Neuron 28, 955-966 (2000).

56. Torborg, C. L., Hansen, K. A. \& Feller, M. B. High frequency, synchronized bursting drives eye-specific segregation of retinogeniculate projections. Nat. Neurosci. 8, 72-78 (2005).

57. Butts, D. A., Kanold, P. O. \& Shatz, C. J. A burst-based 'Hebbian' learning rule at retinogeniculate synapses links retinal waves to activity-dependent refinement. PLoS Biol. 5, e61 (2007).

58. Dilger, E. K. et al. Absence of plateau potentials in dLGN cells leads to a breakdown in retinogeniculate refinement. J. Neurosci. 35, 3652-3662 (2015).

59. Simon, D. K., Prusky, G. T., O'Leary, D. D. \& Constantine-Paton, M. N-methyl$\mathrm{D}$-aspartate receptor antagonists disrupt the formation of a mammalian neural map. Proc. Natl Acad. Sci. USA 89, 10593-10597 (1992).

60. Fagiolini, M. \& Hensch, T. K. Inhibitory threshold for critical-period activation in primary visual cortex. Nature 404, 183-186 (2000).

61. Hensch, T. K. \& Stryker, M. P. Columnar architecture sculpted by GABA circuits in developing cat visual cortex. Science 303, 1678-1681 (2004).

62. Arcelli, P., Frassoni, C., Regondi, M. C., De Biasi, S. \& Spreafico, R. GABAergic neurons in mammalian thalamus: a marker of thalamic complexity? Brain Res. Bull. 42, 27-37 (1997).

63. Rovo, Z. et al. Phasic, nonsynaptic GABA-A receptor-mediated inhibition entrains thalamocortical oscillations. J. Neurosci. 34, 7137-7147 (2014).

64. Guler, A. D. et al. Melanopsin cells are the principal conduits for rod-cone input to non-image-forming vision. Nature 453, 102-105 (2008).

65. Farley, F. W., Soriano, P., Steffen, L. S. \& Dymecki, S. M. Widespread recombinase expression using FLPeR (flipper) mice. Genesis 28, 106-110 (2000).

66. Crone, S. A. et al. Genetic ablation of V2a ipsilateral interneurons disrupts leftright locomotor coordination in mammalian spinal cord. Neuron 60, 70-83 (2008).

\section{Acknowledgements}

We are grateful to Oscar Marin and Lynette Lim at the MRC Centre for Developmental Neurobiology, King's College London for their technical help with live time-lapse imaging, to Diana Garofalo and Dina Balderes from the Sussel lab for providing Nkx2.2 $2^{\text {cre }}$;R26-SLS-tdTomato brains and to Anna Kirjavainen for perfusion fixation of En1 ${ }^{\text {cre }}$;Gata2 $2^{f l x}$ brains used in this study. This research was funded by BBSRC Grant BB/ L020068/1 to A.D. and a Wellcome Trust Grant WT094211MA to S.G.B. and W.W.

\section{Author contributions}

All authors contributed extensively to the work discussing the results and commenting on the manuscript at all stages. A.D. and S.G.B. instigated this work and wrote the paper P.J., Z.Y. and A.D. performed the experiments and analysed data; X.Y., J.P. and L.Z. provided additional materials.

\section{Additional information}

Supplementary Information accompanies this paper at http://www.nature.com/ naturecommunications

Competing financial interests: The authors declare no competing financial interests

Reprints and permission information is available online at http://npg.nature.com/ reprintsandpermissions/

How to cite this article: Jager, P. et al. Tectal-derived interneurons contribute to phasic and tonic inhibition in the visual thalamus. Nat. Commun. 7, 13579 doi: 10.1038/ncomms13579 (2016).

Publisher's note: Springer Nature remains neutral with regard to jurisdictional claims in published maps and institutional affiliations.

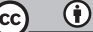

This work is licensed under a Creative Commons Attribution 4.0 International License. The images or other third party material in this article are included in the article's Creative Commons license, unless indicated otherwise in the credit line; if the material is not included under the Creative Commons license, users will need to obtain permission from the license holder to reproduce the material. To view a copy of this license, visit http://creativecommons.org/licenses/by/4.0/

C) The Author(s) 2016 